\title{
Tecnologías de información y comunicación (TIC) aplicadas al tratamiento de los problemas de ansiedad
}

\section{Information and Communication Technologies (ICT) Applied to the Treatment of Anxiety Problems}

\author{
Natalia Regidor y Berta Ausín \\ Universidad Complutense de Madrid, España
}

\begin{abstract}
Resumen: El objetivo general de este artículo es revisar los estudios publicados entre enero de 2008 y diciembre de 2018 acerca del uso y la eficacia de las intervenciones que emplean las TIC en el tratamiento de los problemas de ansiedad. Se revisan 22 estudios y se describen los diferentes formatos de aplicación de las TIC. Aunque los estudios revisados parecen reducir de manera significativa los síntomas de ansiedad hay que señalar que los 22 estudios difieren en aspectos tales como el número y edad de los participantes, el tipo de trastorno de ansiedad objeto de estudio, el tipo de intervención (basada en una App, internet, realidad virtual, etc.), el diseño o las variables de cambio e instrumentos de evaluación elegidos. Todas estas diferencias dificultan poder extraer conclusiones sólidas acerca de la eficacia del uso de las TIC en el tratamiento de los problemas de ansiedad.

Palabras claves: tecnología de la información y comunicación (TIC), Trastorno de ansiedad, eficacia, aplicaciones móviles, mHealth
\end{abstract}

\begin{abstract}
The overall objective of this article is to review the studies published between January 2008 and December 2018 on the use and efficacy of interventions that use ICT in the treatment of anxiety problems. A total of 22 studies are reviewed, and different forms of ICT implementation are described. Although the studies reviewed appear to indicate a significant reduction in anxiety symptoms, it should be noted that the 22 studies differ in aspects like the
\end{abstract}

Berta Ausin (iD https://orcid.org/0000-0001-9497-0275 es Docente en el Departamento de Personalidad y Evaluación Psicólogica en la Universidad Complutense de Madrid. España.

Natalia Regidor, es Psicóloga en CES EDUCA. Universidad Complutense de Madrid. España.

La correspondencia de este artículo debe ser enviada a Berta Ausín al email: bausin@ucm.es

(cc) BY-NC-ND Este es un artículo Open Access bajo la licencia CC BY-NC-ND. 
number and age of the participants, type of anxiety disorder under study, type of intervention (App, online, virtual reality, etc.), design and outcome variables and assessment instruments chosen. All these differences make it difficult to draw strong conclusions regarding the efficacy of the use of ICT in the treatment of anxiety problems.

Keywords: information and communication technology (ICT), Anxiety disorder, efficacy, mobile apps, mHealth

En el ámbito de la salud se han venido empleando en gran medida las tecnologías de información y comunicación (TIC) (Suriá Martínez y Beléndez, 2011). Entre estas tecnologías aplicadas a la salud, las aplicaciones para móvil (apps) surgieron a finales del año 2008, y aproximadamente 40.000 estuvieron relacionadas con el ámbito de la salud. De todas estas apps, el $18 \%$ van dirigidas al control y autogestión del sueño y el estrés, facilitando sobre todo herramientas de relajación (Sierra et al., 2016). El uso de las TIC en psicología se basa en una intervención con los mismos principios psicológicos que la intervención presencial, y consiste en aplicar los mismos tratamientos cognitivo-conductuales mediante un formato diferente (Bornas et al., 2002).

Autores como Simon y Ludman (2009), categorizan las nuevas tecnologías según el grado de contacto humano. En el nivel más alto sitúan aquellas que suponen una comunicación directa con el terapeuta, como la psicoterapia telefónica o videoconferencia. En el nivel intermedio, figuran las sincrónicas, con mensajes de texto que aparecen en función de la presencia de determinados síntomas y que permiten pasar a un nivel máximo de interacción (contactando directamente con el paciente). O bien comunicación de mínimo nivel, de tipo asincrónico como mensajes de texto con recordatorios de fármacos, pero de forma anónima.

Con el fin de conocer el uso de las TIC y las actitudes acerca de este abordaje entre los psicólogos de la Comunidad de Madrid, González-Peña et al. (2017) realizan una encuesta a través del Colegio Oficial de la Psicología de Madrid. Encontraron que un 26\% de las personas encuestadas utilizaba la teleterapia. Los resultados informan de que el $26.66 \%$ utilizaban en el momento de la encuesta terapia por videoconferencia; y el $51.04 \%$ utilizaban Internet como apoyo a la terapia tradicional. Respecto a los aspectos que preocupaban a los psicólogos encuestados, se observó que el $28.29 \%$ afirmaban que en la terapia online se perdía información relevante para el proceso de evaluación / intervención, mientras que un $28.04 \%$ informaban de la dificultad de lograr un buen rapport con el uso de las TIC. Se observa que el $49.18 \%$ de los encuestados están muy interesados en conocer las directrices de la terapia online, y el $45.27 \%$ afirman necesitar información sobre qué tipo de personas y problemáticas se benefician más con este tipo de abordaje.

En cuanto a los formatos de aplicación de las TIC al ámbito de la psicología, la telepsicología engloba toda actividad en el ámbito de la psicología a distancia, apoyándose en la tecnología (teléfono, e-mail, Internet, redes sociales y aplicaciones móvil) (Botella et al., 2007). La telepsicología incluye la teleterapia, que se divide en terapia a distancia, terapia en web con programas automatizados y terapia virtual con simulación de ambientes con o sin terapeuta virtual, que guía el proceso terapéutico del paciente (Botella et al., 2007). A esto hay que añadir otros formatos como la realidad virtual -gracias a la cuál las personas pueden trabajar en esos escenarios a tiempo real) - y la realidad aumentada - la cual supone la introducción de elementos virtuales en el mundo real (Botella et al., 2007). A esto hay que añadir otro tipo de recursos online como son las apps, que permiten eliminar las barreras que suponen acudir a tratamiento. Además de esta ventaja del uso de las TIC en el ámbito de la psicología, diferentes estudios han encontrados que las TIC pueden mejorar la eficacia de algunos tratamientos (Boschen y Casey, 2008), aumentar el compromiso por parte del paciente hacia el tratamiento (Brezinka, 2008), generalizando los principios terapéuticos aprendidos a su vida cotidiana (Clough y Casey, 2011), potenciando el mantenimiento de los logros tras la intervención psicológica (Clough y Casey, 2011), y mejorado la calidad de vida de las personas durante y tras la intervención (Bornas et al., 2002).

Este artículo se centra en el uso de las TIC en el tratamiento de los trastornos de ansiedad por varios motivos. Por un lado, se trata de uno de los problemas de salud mental con mayores tasas de prevalencia. Según el estudio europeo ESEMeD-España realizado con población general, la prevalencia anual de este trastorno es del $10.6 \%$ y la prevalencia vital del $16.6 \%$ (Haro et al., 2006). Las tasas de prevalencia de los trastornos de 
ansiedad en las personas mayores de 65 años europeas en el último año van desde un $3.6 \%$ (Alonso et al, 2004) hasta un 17.2\% (Andreas et al., 2017) en la revisión de 36 estudios realiza por Cisneros y Ausín (2019). Por otro lado, los trastornos de ansiedad conllevan una disminución en el funcionamiento de las personas que los sufren y una peor calidad de vida (Canuto et al., 2018; Zamorano et al., 2019). Por último, es relevante estudiar el uso de las TIC como complemento en el tratamiento de los trastornos de ansiedad ya en los últimos años se viene observando que el $25 \%$ de los pacientes con un trastorno de ansiedad rechazan la técnica de exposición y abandonan el tratamiento (Baños et al., 2013).

El objetivo general de este artículo es revisar los estudios publicados entre enero de 2008 y diciembre de 2018 acerca del uso y la eficacia de las intervenciones que emplean las TIC en el tratamiento de los problemas de ansiedad. Se pretende encontrar respuesta a las siguientes preguntas. En cuanto a los participantes $i s o n$ eficaces las intervenciones que emplean las TIC en el tratamiento de los problemas de ansiedad en personas de cualquier edad? En cuanto a las intervenciones ¿cuáles son las TIC que se emplean en el tratamiento de los problemas de ansiedad? Respecto a las comparaciones ¿qué componentes incluyen las intervenciones que emplean las TIC en el tratamiento de los problemas de ansiedad? En cuanto a los resultados, cabe preguntarse: de las intervenciones que emplean las TIC en el tratamiento de los problemas de ansiedad ¿cuáles son eficaces? Respecto al diseño de los estudios ¿siguen una metodología de ensayo clínico randomizado?

\section{Método}

Se realiza una revisión de la literatura científica sobre programas de intervención con el uso de las TIC como parte central o complementaria en el tratamiento de los trastornos de ansiedad en población general. Se consultan las bases de datos PsycInfo, Psycarticle y Medline, y el motor de búsqueda Pubmed. Las palabras clave utilizadas son: "m-Health" "anxiety disorders", "mobiles apps" "ICTs; Psychology 2.0", "Health 2.0", "ePatients", "smartphone", "treatments", "technological Internet" y "virtual reality".

Los criterios de inclusión empleados fueron:

- Artículos escritos en español e inglés.

- Artículos publicados de enero 2008 - diciembre 2018.

- Estudios de intervenciones en problemas de ansiedad a través del uso de las TIC.

Tabla 1. Número de artículos encontrados y eliminados según base de datos y otras fuentes

\begin{tabular}{|c|c|c|c|c|c|}
\hline $\begin{array}{l}\text { BASES DE } \\
\text { DATOS }\end{array}$ & $\begin{array}{l}\mathbf{N}^{\circ} \text { Artículos } \\
\text { encontrados }\end{array}$ & $\begin{array}{c}\mathbf{N}^{\mathbf{o}} \\
\text { Artículos } \\
\text { eliminados }\end{array}$ & $\begin{array}{c}\mathrm{N}^{\mathbf{o}} \\
\text { Artículos } \\
\text { elegidos }\end{array}$ & $\begin{array}{l}\stackrel{\mathrm{N}^{\mathbf{O}}}{\mathrm{Artículos}} \\
\text { repetidos }\end{array}$ & $\begin{array}{c}\mathbf{N}^{\mathbf{0}} \\
\text { Artículos } \\
\text { finales }\end{array}$ \\
\hline Pubmed & 168 & 142 & 26 & \multirow{5}{*}{35} & \multirow{5}{*}{22} \\
\hline PsycInfo & 116 & 102 & 14 & & \\
\hline Psycarticle & 70 & 69 & 1 & & \\
\hline Medline & 102 & 90 & 12 & & \\
\hline $\begin{array}{l}\text { Búsqueda por } \\
\text { otras fuentes }\end{array}$ & 27 & 23 & 4 & & \\
\hline TOTAL & 489 & 426 & 57 & 35 & 22 \\
\hline
\end{tabular}


Los criterios de exclusión fueron, en consecuencia, no cumplir con los criterios de inclusión establecidos.

De los resultados de la búsqueda bibliográfica se identificaron 435 artículos de las bases de datos y 54 citas adicionales identificados en otras fuentes. De los cuales 35 fueron descartados por aparecer duplicados. A partir de la lectura del título y del abstract se excluyeron 315 artículos por tener un objetivo diferente al de la presente revisión. Además, se descartaron 2 estudios por estar publicados en un idioma distinto al castellano o al inglés. Por otro lado, en el árbol de búsqueda presentado en la figura 1 se observa que 117 estudios se descartaron por no incluir una intervención a través de las TIC para el trastorno de ansiedad. Finalmente se seleccionaron 22 estudios que cumplían con todos los criterios de inclusión establecidos (tabla 1).

Figura 1. Diagrama de flujo de la búsqueda bibliográfica

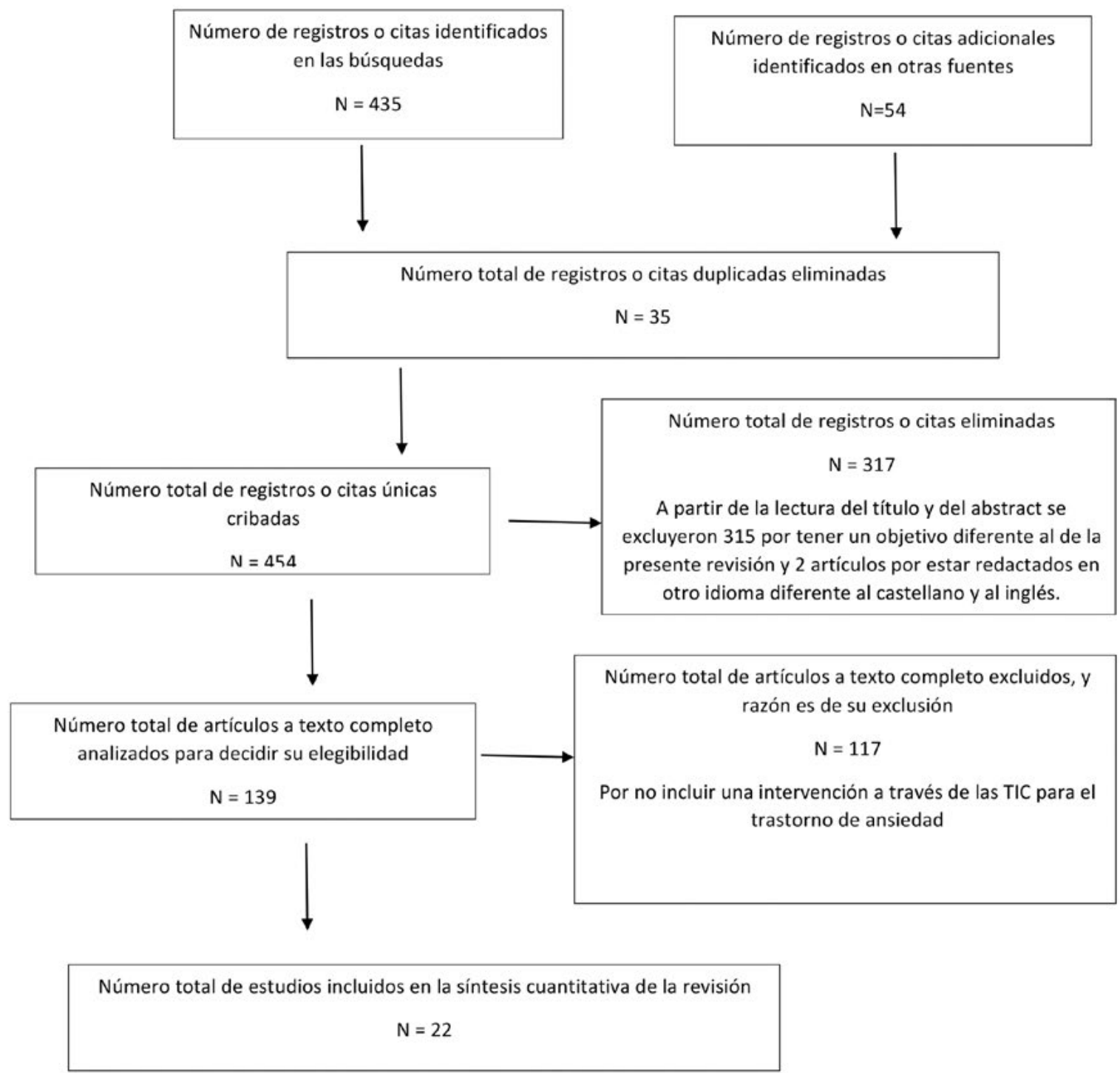

\section{Resultados}

En la tabla 2, al final del artículo, se recoge la descripción de los 22 estudios incluidos en esta revisión. La tabla 3, ver final del artículo, describe brevemente cada uno de los programas de intervención aplicados.

En cuanto a los países en los que se han realizado los diferentes estudios, como se observa en la tabla 2, de los 22 estudios totales, 5 de ellos se llevaron a cabo en Suecia (Ekberg et al., 2011; Hedman et al., 2013; 2011; 
Lindner et al., 2013; Ly et al., 2012), 4 estudios se llevaron a cabo en diferentes estados de los Estados Unidos (Gary y Amresh, 2017; Mohr et al., 2017; Pramana et al., 2018; Pramana et al., 2014), 8 estudios se realizaron en Australia (Spence et al., 2011; Christensen et al., 2014; Proudfoot et al., 2013; Spence et al., 2011) Italia (Grassi et al., 2009; Pallavicini et al., 2009; Villani et al., 2013) y Reino Unido (Christoforou et al., 2017; Pham et al., 2016). Los 5 estudios restantes se realizaron en Suiza, Canadá, España, Rumanía y Dublín.

En relación a las muestras empleadas, sumando las muestras de los 22 estudios revisados, la muestra de sujetos asciende a 2.979 personas, que han participado en diversos programas de intervención para reducir los síntomas de ansiedad, que contenían como parte principal o secundaria, la intervención con TIC. El estudio con menor muestra cuenta con 5 sujetos (Benta et al., 2015), mientras que el estudio con mayor número de sujetos tiene 720 (Proudfoot et al., 2013). Las personas que han participado en los estudios analizados presentaban los siguiente trastornos de ansiedad: Trastorno de Ansiedad Generalizada (Christensen et al., 2014; Ekberg et al., 2011; Gary y Amresh, 2017; Grassi et al., 2009; Hedman et al., 2013; Ly et al., 2012; Mohr et al., 2017; Pallavicini et al., 2009; Pramana et al., 2014; 2018; Proudfoot et al., 2013; Spence et al., 2011; Villani et al., 2013; Wozney et al., 2015), Ansiedad social, Ansiedad por separación (Hedman et al., 2011; Linder et al., 2013; Per Carlbring et al., 2007; Pramana et al., 2014; 2018; Spence et al., 2011; Stolz et al., 2018) Fobia específica (Campos et al., 2016; Spence et al., 2011), Agorafobia (Christoforou et al., 2017) Trastorno de pánico (Linder et al., 2013; Pham et al., 2016).

Todos los estudios que se han tenido en cuenta incluyen tantos sujetos menores de 18 años (con el consentimiento y acompañamiento de sus progenitores) o mayores de 18, pero ningún estudio cuenta con una muestra de edad superior a 50 años.

Los 22 estudios seleccionados indican tanto el tamaño de la muestra como el rango de edad de la misma. Además, cada estudio tiene sus propios criterios de inclusión. En este sentido, los 22 estudios incluyen como criterio de inclusión presentar algún tipo de trastorno de ansiedad y, como criterio de exclusión, acudir en ese momento a algún tipo de terapia o haber acudido anteriormente a terapia psicológica.

En cuanto al tiempo empleado, cada tipo de intervención tiene su duración. Los tiempos van desde una semana de intervención (Benta et al., 2015) hasta los 3 meses (Ekberg et al., 2011), aunque la mayoría de las intervenciones, tienen una duración de entre 4-12 semanas. La duración de las sesiones oscila entre 10 minutos (Grassi et al., 2009) y 60 minutos (Spence et al., 2011). Cinco intervenciones especifican su duración (Ekberg et al., 2011; Gary y Amresh, 2017; Grassi et al., 2009; Hedman et al., 2011; Spence et al., 2011), mientras que los 17 estudios restantes no indican la duración de las sesiones, únicamente el número y la cantidad de sesiones por semanas. Respecto a las medidas PRE, POST y el seguimiento, 12 estudios incluyen las tres medidas, mientras que 10 solo han medido las variables deseadas PRE y POST intervención. De todos estos estudios 18 incluyen grupo experimental y grupo control, el resto de los estudios no especifican los grupos formados. En la tabla 2 se observa que 11 de los estudios siguen una metodología de ensayo clínico randomizado y 11 estudios no. Todos los estudios revisados son estudios cuasiexperimentales, ya que no hay control absoluto de todas sus variables, y puede que los resultados estén afectados por otras variables extrañas que no han sido controladas. Los estudios miden diferentes constructos, algunos se centran en los efectos de la intervención sobre los síntomas de ansiedad, mientras que otros centran su interés en el impacto de la intervención sobre la calidad de vida. Por último, otros estudios valoran estas dos variables. Además, no se utilizan los mismos instrumentos de medida para el mismo constructo en los diferentes estudios.

En los estudios analizados se han obtenido diferentes resultados de eficacia de las intervenciones según el tipo de trastorno que padecía la muestra de sujetos. Los sujetos con Trastorno por ansiedad social en los estudios de Benta et al. (2015), Hedman et al. (2011), Per Carlbring et al. (2007) y Stolz et al. (2018) mostraron mejoras significativas en ansiedad social, miedo y evitación haciendo uso de las TIC. Estas personas mostraban un alto bienestar y al final de la intervención no cumplían con el diagnóstico de ansiedad social. En los sujetos con Fobias específicas de los estudios de Campos et al. (2016) y Spence et al. (2011) mostraron beneficios significativos, además de una reducción de la ansiedad y obtuvieron mayores mejorías en el funcionamiento general. Además, se superaron las barreras que limitan a los sujetos a exponerse generando una mayor adherencia y menor tasa de abandonos. En los sujetos con Trastorno de Ansiedad Generalizada de los estudios de Christensen et al. (2014), Ekberg et al. (2011), Gary y Amresh (2017), Grassi et al. (2009) Hedman et al. (2013), Ly et al. 
(2012), Mohr et al. (2017), Pallavicini et al. (2009) Pramana et al. (2014), Proudfoot et al. (2013), Villani et al. (2013) y Wozney et al. (2015) mostraron preferencias por el uso de las TIC en las intervenciones, produciendo altos niveles de satisfacción. Se mostraron reducciones significativas en los niveles de ansiedad, así como un aumento del nivel de relajación tras el uso de las TIC. En la misma línea, el uso de las TIC demostró mejorías moderadas en los niveles de ansiedad y depresión en general. Al final del tratamiento los sujetos cumplieron con los criterios de remisión completa o sin síntomas de ansiedad, además, las mejorías que se obtuvieron durante las intervenciones se mantuvieron en el seguimiento. Estos estudios, señalan que las aplicaciones móviles como intervenciones $m H e a l t h$, pueden ser eficaces en la reducción de los niveles de ansiedad.

En los sujetos con Agorafobia en el estudio de Christoforou et al. (2017) mostraron que al inicio hubo un alto nivel de evitación agorafóbica severa, pero tras el uso de la app "Agoraphobia Free" hubo mejoras estadísticamente significativas en la gravedad de los síntomas. En los sujetos con Trastorno de Pánico de los estudios de Lindner et al. (2013) y Pham et al. (2016) mostraron que los sujetos tuvieron puntuaciones mejoradas en ansiedad, pánico, hiperventilación y calidad de vida, gracias al uso de las apps e Internet. Finalmente, los sujetos con Trastorno de Ansiedad por Separación de los estudios de Pramana et al. (2018) y Spence et al. (2011) mostraron que hubo beneficios significativos, reducciones significativamente mayores en los niveles de ansiedad, así como una mejora en el funcionamiento general. Los sujetos se mostraron más motivados gracias al entorno interactivo y divertido que generan las aplicaciones móviles, haciendo uso de las aplicaciones, y encontrándola útil cuando experimentaban ansiedad.

\section{Discusión y conclusiones}

Uno de los objetivos del presente trabajo fue realizar una revisión de los estudios acerca del uso de las TIC como elemento terapéutico en el tratamiento de los problemas de ansiedad. La mayoría de ellos, no estudiaban los trastornos de ansiedad de manera aislada, sino que lo hacían junto con los trastornos depresivos. Todos los estudios que se han revisado (Campos et al., 2016; Christensen et al., 2014; Christoforou et al., 2017; Ekberg et al., 2011; Gary y Amresh, 2017; Grassi et al., 2009; Hedman et al., 2011; 2013; Linder et al., 2013; Ly et al., 2012; Mohr et al., 2017; Pallavicini et al., 2009; Pham et al., 2016; Per Carlbring et al., 2007; Pramana et al., 2014; 2018; Proudfoot et al., 2013; Spence et al., 2011; Stolz et al., 2018; Villani et al., 2013; Wozney et al., 2015) han utilizado las TIC como parte complementaria al tratamiento tradicional, e incluso en algunos casos las TIC tenían mayor visibilidad, siendo la parte central y clave del tratamiento.

Con esta revisión se buscaba encontrar respuesta a algunas preguntas. En cuanto a los participantes nos preguntábamos si son eficaces las intervenciones que emplean las TIC en el tratamiento de los problemas de ansiedad en personas de cualquier edad. Los estudios revisados no incluyen personas mayores de 50 años, por lo que los resultados no son generalizables a toda la población. De los 22 estudios sólo 5 de ellos incluían a menores de edad. Son necesarios estudios en este sentido con población menor de edad y mayor de 50 años.

En cuanto a las intervenciones pretendíamos conocer cuáles son las TIC que se emplean en el tratamiento de los problemas de ansiedad. Esta revisión ha puesto de relieve que son muy variados los tipos de TIC que se han empleado en el tratamiento de los problemas de ansiedad, que van desde un asistente a través de móvil, tratamiento basado en internet, a diferentes Apps. Respecto a las comparaciones entre los diferentes estudios analizados nos preguntábamos al inicio por los componentes que incluyen las intervenciones que emplean las TIC en el tratamiento de los problemas de ansiedad. Como se ha descrito en la tabla 2, los 22 estudios difieren en muchos aspectos: número y edad de los participantes, tipo de trastorno de ansiedad objeto de estudio, tipo de intervención (basada en una App, internet, realidad virtual, etc.), diseño, variables de cambio e instrumentos de evaluación elegidos, entre otros. Todas estas diferencias dificultan poder extraer conclusiones sólidas acerca de la eficacia del uso de las TIC en el tratamiento de los problemas de ansiedad.

En la introducción de este artículo nos preguntábamos: de las intervenciones que emplean las TIC en el tratamiento de los problemas de ansiedad ¿cuáles son eficaces? Teniendo en cuenta las limitaciones de los estudios antes descritas, se puede concluir que los resultados obtenidos en la presente revisión indica que el uso de las TIC en terapia, como herramienta terapéutica, puede ser un componente eficaz para reducir la sintomatología 
ansiosa. En este sentido, los resultados de los estudios revisados apuntan a mejoras en los síntomas de ansiedad empleando intervenciones basadas en las TIC (Campos et al., 2016; Christensen et al., 2014; Christoforou et al., 2017; Ekberg et al., 2011; Gary y Amresh, 2017; Grassi et al., 2009; Hedman et al., 2011; 2013; Linder et al., 2013; Ly et al., 2012; Mohr et al., 2017; Pallavicini et al., 2009; Pham et al., 2016; Per Carlbring et al., 2007; Pramana et al., 2014; 2018; Proudfoot et al., 2013; Spence et al., 2011; Stolz et al., 2018; Villani et al., 2013; Wozney et al., 2015). Por otro lado, la mayoría de los estudios muestran que las aplicaciones de prevención e intervención en los problemas de ansiedad son valoradas positivamente por los usuarios. Éstos se muestran más motivados y con más probabilidades de participar en el aprendizaje de habilidades de terapia cognitivo-conductual (TCC) utilizando un entorno de aprendizaje interactivo, como son los juegos. Gracias a las TIC los estudios muestran una disminución significativa del nivel de ansiedad en el tiempo, así como eficacia, para mejorar su bienestar en situaciones de estrés de la vida cotidiana. Uno de los estudios de la revisión (Pramana, et al., 2014) muestra incluso que la aplicación "SmartCAT puede integrarse con éxito en la TCC con niños con trastornos de ansiedad.

Respecto al diseño de los estudios nos preguntábamos si seguían una metodología de ensayo clínico randomizado y encontramos que 11 de los estudios siguen una metodología de ensayo clínico randomizado y 11 estudios no. Una vez más hay que tomar con cautela los resultados de esta revisión, ya que la mitad de los estudios no cumplen los criterios de calidad en este sentido.

Por otro lado, algunos estudios se han llevado a cabo en una muestra de personas que presentaban únicamente algún trastorno de ansiedad, en cambio, otros estudios muestran que los usuarios presentan otro tipo de trastorno como es el caso de la depresión (Proudfoot et al., 2013; Mohr et al. 2017; Ly et al., 2012). La ventaja de que los estudios únicamente presenten el trastorno de ansiedad es que el tratamiento utilizado se puede adaptar de manera más concreta y mejor al trastorno que se está tratando.

Todos los estudios se han realizado de manera individual (previa distribución al azar según la condición de tratamiento) (Campos et al., 2016; Christensen et al., 2014; Christoforou et al., 2017; Ekberg et al., 2011; Gary y Amresh, 2017; Grassi et al., 2009; Hedman et al., 2011; 2013; Linder et al., 2013; Ly et al., 2012; Mohr et al., 2017; Pallavicini et al., 2009; Pham et al., 2016; Per Carlbring et al., 2007; Pramana et al., 2014; 2017; Proudfoot et al., 2013; Spence et al., 2011; Stolz et al., 2018; Villani et al., 2013; Wozney et al., 2015). El periodo temporal de las intervenciones realizadas en los diferentes estudios, no parece que influya en los resultados, ya que cada uno de los programas tiene efectos similares, mostrando resultados favorecedores para el uso de las TIC en el tratamiento de la ansiedad. En la mayoría de los casos hay mejoras a nivel de salud psicológica (remisión completa o sin síntomas de ansiedad) y física, además de un mejor funcionamiento general, manteniéndose los efectos en el seguimiento. Los criterios de inclusión de cada estudio son razonables teniendo en cuenta la muestra con la que se quiere trabajar y, aunque la mayoría no cuentan con una gran cantidad de requisitos, uno de ellos es fundamental: presentar diagnóstico de trastorno de ansiedad y no haber recibido terapia psicológica anteriormente, lo que hace que limite la muestra lo suficiente.

De la revisión realizada se concluye que es muy importante que los profesionales que lleven a cabo intervenciones para reducir los síntomas de ansiedad desde la telepsicología estén bien formados y especializados en el uso de las TIC. En la mayoría de los estudios revisados se hace explícito que el terapeuta está bien cualificado para realizar la intervención, además de incluir a otros profesionales para la supervisión de los terapeutas. Estos profesionales también llevan a cabo el seguimiento de los usuarios, obteniendo así resultados muy positivos en todos los casos.

El avance de las TIC aplicadas a la evaluación e intervención psicologica es un hecho que está aumentando de manera progresiva. Entre las ventajas del empleo de las TIC en este contexto nos encontramos con que permiten aumentar la motivación al cambio en los usuarios, permite una mayor accesibilidad a los recursos de salud, y facilita una mayor percepción de control en el caso de la técnica de exposición, pudiendo personalizar cada escenario virtual a las necesidades de la persona. Estas herramientas nos ofrecen asistencia para realizar las tareas, así como obtener un feedback de los posibles riesgos que los usuarios pueden experimentar, o adquirir y entrenar nuestras capacidades a través de actividades interactivas gracias a las aplicaciones.

Sin embargo, existen limitaciones que tenemos que tener en cuenta, como puede ser la incertidumbre del profesional que hay detrás “¿quién se encuentra al otro lado?”. Importante destacar una de las mayores limi- 
taciones que podemos encontrarnos y que es clave en la terapia psicológica, la relación terapéutica. En este sentido, las nuevas tecnologías pueden suponer una barrera a la hora de realizar terapia, ya que pueden llevar a una falta de espontaneidad en la relación terapeuta-paciente. Otro de los grandes obstáculos que presenta el uso de las TIC son los costes económicos, en el caso concreto de la realidad virtual y la realidad aumentada, suponen un coste elevado, que muchos profesionales e incluso instituciones no pueden asumir, aumentando el rechazo hacia su uso por parte de los profesionales, ya que, aunque los datos muestren mejoras significativas, no están al alcance de muchos.

A pesar de ello, haciendo un balance entre las aportaciones de las nuevas tecnologías y sus limitaciones, es evidente que los beneficios superan a las limitaciones, ya que garantizan una mejora en la terapia y en la salud de los pacientes, haciendo que, concretamente en el caso de los trastornos de ansiedad, aumente la calidad de vida del paciente. A pesar de las dificultades, es inevitable la eficacia prometedora del uso de aplicaciones móviles o las terapias basadas en Internet como intervenciones de mHealth y que resultarán un indudable y prometedor futuro.

A pesar de las ventajas del uso de las TIC en el contexto clínico, se sabe que este tipo de herramientas tiene un uso reducido en la terapia psicológica, por lo que es necesario en línea futuras seguir realizando investigaciones, con el fin de comprobar si la adopción de las TIC será satisfactoria para todos los grupos de edad, para los diferentes países, diferentes tipos de trastornos o incluso para el uso de las TIC por parte de los familiares ya que estos pueden identificar las señales de riesgo de manera más rápida. Sin duda estas investigaciones tienen que ir con un fin concreto, como es el aumento en la calidad de vida de las personas.

Para poder tener una visión óptima hacia el futuro será necesario estudiar la eficacia de las TIC en la intervención con niños y personas mayores, adaptar las apps existentes a las personas mayores, niños, personas con alguna discapacidad y estudiar la eficacia de las aplicaciones actuales. Una vez hecho esto, será necesario traducir al castellano estas aplicaciones y realizar cursos de formación a los profesionales de la salud en el uso de las TIC, además de incluir esta forma de intervención en los programas de formación universitaria en Psicología tanto en la evaluación como en el tratamiento de los problemas de ansiedad. Por otro lado, es importante mejorar las garantías éticas y científicas del uso de las TIC en el tratamiento de los problemas de salud mental, así como mejorar la garantía de los derechos de protección de datos en el uso de las TIC en la evaluación y tratamiento de los problemas de ansiedad. Finalmente, sería conveniente incluir en los códigos deontológicos de la profesión del psicólogo aspectos relacionados con la evaluación y tratamiento de los problemas de ansiedad a través de las TIC.

Finalmente, debido al gran potencial que muestran las TIC en el ámbito de la psicología clínica y que seguirán siéndolo en un futuro, es clave dar a conocer a los profesionales de la salud todas las oportunidades que ofrecen para el tratamiento de los diferentes tipos de trastornos, así como una continua investigación para aumentar el número de tratamientos y tipos de trastornos a los que se dirigen. Un buen ejemplo de esfuerzo en este sentido es la reciente publicación de la Guía para la práctica de la telepsicología publicada por el Consejo General de la Psicología en España, y la Guía para la intervención Telepsicológica del Colegio Oficial de la Psicología de Madrid. En el futuro deberemos adecuar cada vez más nuestro sistema sanitario para dar sustento a los recursos de telepsicología.

\section{Referencias}

Andreas, S., Schulz, H., Volkert, J., Dehoust, M., Sehner, S., Suling, A., Ausín, B., Canuto, A., Crawford, M.,

Da Ronch, C., Grassi, L., Hershkovitz, Y., Muñoz, M., Quirk, A., Rotenstein, O., Santos-Olmo, A. B., Shalev, A., Strehle, J., Weber, K., ... Härter, M. (2017). Prevalence of mental disorders in elderly people: the European MentDis_ICF65+ study. The British Journal of Psychiatry, 210(2), 125-131. https://doi.org/10.1192/ bjp.bp. 115.180463

Alonso, J., Angermeyer, M. C., Bernert, S., Bruffaerts, R., Brugha, T. S., Bryson, H., de Girolamo, G., Graaf, R., Demyttenaere, K., Gasquet, I., Haro, J. M., Katz, S. J., Kessler, R. C., Kovess, V., Lépine, J. P., Ormel, J., Polidori, G., Russo L. J., Vilagut, G., ... Vollebergh, W. A. (2004). Prevalence of mental disorders in Eu- 
rope: Results from the European Study of the Epidemiology of Mental Disorders (ESEMeD) project. Acta Psychiatrica Scandinavica, 109(s420), 21-27. https://doi.org/10.1111/j.1600-0047.2004.00327.x

Baños, R. M., Guillén, V., García-Palacios, A., Quero, S., y Botella, C. (2013). Las nuevas tecnologías en el tratamiento de los trastornos de ansiedad. Información Psicológica, 102(2) 28-46.

Benta, K.-I., Cremene, M., Matu, S.-A. y Podina, I. R. (2015). Speakeasy-a Mobile Assistant for the Management of Social Anxiety in Speech Situations. Acta Technica Napocensis, 56(3), 29-35.

Botella, C., Baños, R. M., García, A., Quero, S., Guillén, V. y Marco, H. J. (2007). El uso de las nuevas tecnologías de la información y la comunicación en psicología clínica. UOC Papers: Revista Sobre La Sociedad Del Conocimiento, 4, 32-41. http://uocpapers.uoc.edu/uocpapers/4/dt/esp/botella.pdf

Bornas, X., Rodrigo, T. y Barceló, F. (2002). Las nuevas tecnologías en la terapia cognitivo-conductual: una revisión. Revista Internacional de Psicología Clínica y de la Salud, 2(3), 533-541.

Boschen, M. J. y Casey, L. M. (2008). The use of mobile telephones as adjuncts to cognitive behavioral psychotherapy. Professional Psychology: Research and Practice, 39(5), 546-552. https://doi.org/10.1037/07357028.39 .5 .546

Brezinka,V. (2008). Treasure Hunt - A serious game to support psychotherapeutic treatment of children. Studies in health technology and informatics, 136, 71-76.

Campos, D., Bretón-López, J., Botella, C., Mira, A., Castilla, D., Baños, R. y Quero, S. (2016). An Internet-based treatment for flying phobia (NO-FEAR Airlines): Study protocol for a randomized controlled trial. Biomedcentral Psychiatry, 16(1), Artículo e296. https://doi.org/10.1186/s12888-016-0996-1

Canuto, A., Weber, K., Baertschi, M., Andreas, S., Volkert, J., Dehoust, M., Sehner, S., Suling, A., Wegscheider, K., Ausín, A., Crawford, M., Ronch, C., Grassi, L., Hershkovitz, Y., Muñoz, M., Quirk, A., Rotenstein, O., Santos-Olmo, A.B., Shalev, A., ... Härter, M. (2018). Anxiety disorders in old age: Psychiatric comorbidities, quality of life, and prevalence according to age, gender, and country. American Journal of Geriatric Psychiatry, 26(2), 174-185. https://doi.org/10.1016/j.jagp.2017.08.015

Christensen, H., Batterham, P., Mackinnon, A., Griffiths, K. M., Kalia Hehir, K., Kenardy, J. y Bennett, K. (2014). Prevention of generalized anxiety disorder using a web intervention, iChill: randomized controlled trial. Journal of Medical Internet Research, 16(9), 199. https://doi.org/10.2196/jmir.3507

Christoforou, M., Sáez Fonseca, J. A. y Tsakanikos, E. (2017). Two Novel Cognitive Behavioral Therapy-Based Mobile Apps for Agoraphobia: Randomized Controlled Trial. Journal of Medical Internet Research, 19(11), 398. https://doi.org/10.2196/jmir.7747

Cisneros, G. E. y Ausín, B. (2019). Prevalencia de los trastornos de ansiedad en las personas mayores de 65 años: una revisión sistemática. Revista Española de Geriatría y Gerontología, 54(1), 34-48. https://doi. org/10.1016/j.regg.2018.05.009

Clough, B. A. y Casey, L. M. (2011). Technological adjuncts to enhance current psychotherapy practices: a review. Clinical Psychology Review, 31(3), 279-292. https://doi.org/10.1016/j.cpr.2010.12.008

Ekberg, J., Timpka, T., Bång, M., Fröberg, A., Halje, K. y Eriksson, H. (2011). Cell phone-supported cognitive behavioural therapy for anxiety disorders: a protocol for effectiveness studies in frontline settings. Biomedcentral Medical Research Methodology, 11(1), Artículo e3. https://doi.org/10.1186/1471-2288-11-3

Gary, K. y Amresh, A. (2017). Usability of a Smartphone Application to Support the Prevention and Early Intervention of Anxiety in Youth. Cognitive and Behavioral Practice 24(4), 393-404. https://doi.org/10.1016/j. cbpra.2016.11.002

González-Peña, P., Torres, R., Barrio, V. D. y Olmedo, M. (2017). Uso de las nuevas tecnologías por parte de los psicólogos españoles y sus necesidades. Clínica y Salud, 28(2), 81-91. https://doi.org/10.1016/j. clysa.2017.01.001

Grassi, A., Gaggioli, A. y Riva, G. (2009). The Green Valley: The Use of Mobile Narratives for Reducing Stress in Commuters. CyberPsychology \& Behavior, 12(2), 155-161. https://doi.org/10.1089/cpb.2008.0156 Haro, J. M., Palacín, C., Vilagut, G., Martínez, M., Bernal, M., Luque, I., Codony, M., Dolz, M., Alonso, J. y el Grupo ESEMeD-España. (2006). Prevalencia de los trastornos mentales y factores asociados: resultados del estudio ESEMeD-España. Medicina Clínica, 126(12), 445-451. https://doi.org/10.1157/13086324 
Hedman, E., Andersson, E., Andersson, G., Lindefors, N., Lekander, M., Rück, C. y Ljótsson, B. (2013). Mediators in internet-based cognitive behavior therapy for severe health anxiety. PloS One, 8(10), Artículo e77752. https://doi.org/10.1371/journal.pone.0077752

Hedman, E., Andersson, G., Ljótsson, B., Andersson, E., Rück, C., Mörtberg, E. y Lindefors, N. (2011). Internet-based cognitive behavior therapy vs. cognitive behavioral group therapy for social anxiety disorder: a randomized controlled non-inferiority trial. PloS One, 6(3), Artículo e18001. https://doi.org/ 10.1371/journal. pone.0018001

Lindner, P., Ivanova, E., Ly, K. H., Andersson, G. y Carlbring, P. (2013). Guided and unguided CBT for social anxiety disorder and/or panic disorder via the Internet and a smartphone application: study protocol for a randomised controlled trial. Trials, 14, 437, Artículo e437. https://doi.org/10.1186/1745-6215-14-437

Ly, K.H., Dahl, J., Carlbring, P. y Andersson, G. (2012). Development and initial evaluation of a smartphone application based on acceptance and commitment therapy. SpringerPlus, 1(1), 11. https://doi. org/10.1186/2193-1801-1-11

Mohr, D. C., Tomasino, K. N., Lattie, E. G., Palac, H. L., Kwasny, M. J., Weingardt, K. y Schueller, S. M. (2017). IntelliCare: An Eclectic, Skills-Based App Suite for the Treatment of Depression and Anxiety. Journal of Medical Internet Research, 19(1), Artículo e10. https://doi.org/10.2196/jmir.6645

Pallavicini, F., Algeri, D., Repetto, C., Gorini, A. y Riva, G. (2009). Biofeedback, virtual reality and mobile phones in the treatment of generalized anxiety disorder (gad): A phase-2 controlled clinical trial. Journal of Cyber Therapy and Rehabilitation, 2(4), 315-327. https://doi.org/10.3233/978-1-60750-561-7-39

Per Carlbring, M., Gunnarsdo T., Linda, H., Gerhard, A., Lisa, E., Tomas, F. (2007). Treatment of social phobia: randomised trial of internet.delivered cognitive .behavioural therapy with telephone support. British Journal of Psychiatry, 190(2), 123-128. https://doi.org/10.1192/bjp.bp.10 5.02 0107

Pham, Q., Khatib, Y., Stansfeld, S., Fox, S. y Green, T. (2016). Feasibility and Efficacy of and mHealth Game for Managing Anxiety: "Flowy" Randomized Controlled Pilot Trial and Design Evaluation. Games for Health Journal, 5(1), 50-67. https://doi.org/10.1089/g4h.2015.0033

Pramana, G., Parmanto, B., Kendall, P. C. y Silk, J. S. (2014). The SmartCAT: an m-health platform for ecological momentary intervention in child anxiety treatment. Telemedicine Journal and E-Health: The Official Journal of the American Telemedicine Association, 20(5), 419-427. https://doi.org/ 10.1089/tmj.2013.0214.

Pramana, G., Parmanto, B., Lomas, J., Lindhiem, O., Kendall, P. C. y Silk, J. (2018). Using Mobile Health Gamification to Facilitate Cognitive Behavioral Therapy Skills Practice in Child Anxiety Treatment: Open Clinical Trial. Journal of Medical Internet Research JMIR Serious Games, 6(2), Artículo e9. https://doi. org/10.2196/games.8902

Proudfoot, J., Clarke, J., Birch, M.-R., Whitton, A. E., Parker, G., Manicavasagar, V., y Hadzi-Pavlovic, D. (2013). Impact of a mobile phone and web program on symptom and functional outcomes for people with mild-to-moderate depression, anxiety and stress: a randomised controlled trial. Biomedcentral BMC Psychiatry, 13(1), Artículo 312. https://doi.org/10.1186/1471-244X-13-312

Sierra, P., Gallach, E., Echevarría, H., García Blanco, A. y Livianos, L. (2016). ¿Qué pueden aportar actualmente las nuevas tecnologías al trastorno bipolar? Revista de Psicopatología y Psicología Clínica, 21(1), 45-56. https://doi.org/ 10.5944/rppc.vol.21.num.1.2016.16373

Simon, G. E. y Ludman, E. J. (2009). It's time for disruptive innovation in psychotherapy. The Lancet, 374(9690), 594-595. https://doi.org/10.1016/S0140-6736(09)61415-X.

Spence, S. H., Donovan, C. L., March, S., Gamble, A., Anderson, R. E., Prosser, S. y Kenardy, J. (2011). A randomized controlled trial of online versus clinic-based CBT for adolescent anxiety. Journal of Consulting and Clinical Psychology, 79(5), 629-642. https://doi.org/10.1037/a0024512

Stolz, T., Schulz, A., Krieger, T., Vincent, A., Urech, A., Moser, C. y Berger, T. (2018). A mobile App for social anxiety disorder: A three-arm randomized controlled trial comparing mobile and PC-based guided selfhelp interventions. Journal of Consulting and Clinical Psychology, 86(6), 493-504. https://doi.org/10.1037/ ccp0000301

Suriá Martínez, R. y Beléndez, M. (2011). Grupos de apoyo virtuales dedicados a problemas de salud: estudio de su tipología y análisis de su representatividad. Anales de Psicología, 27(1), 210-220. 
Villani, D., Grassi, A., Cognetta, C., Toniolo, D., Cipresso, P. y Riva, G. (2013). Self-help stress management training through mobile phones: an experience with oncology nurses. Psychological Services, 10(3), 315322. https://doi.org/ 10.1037/a0026459

Wozney, L., Baxter, P. y Newton, A. S. (2015). Usability evaluation with mental health professionals and young people to develop an Internet-based cognitive-behaviour therapy program for adolescents with anxiety disorders. Biomedicalcentral Pediatrics, 15, Artículo e213. https://doi.org/10.1186/s12887-015-0534-1

Zamorano, A., Munoz, M., Ausin, B. y Perez, E. (2019). Relationship between mental health and level of functioning in people over 65 years in Madrid Region. Clínica y Salud, 30(2), 63-71. https://doi.org/10.5093/ clysa2019a8

Artículo recibido: 01/10/2019

Artículo aceptado: 29/05/2020 
Tabla 2. Descripción de los estudios seleccionados

\begin{tabular}{|c|c|c|c|c|c|c|c|c|c|}
\hline ESTUDIO & $\begin{array}{c}\text { TIPO DE } \\
\text { TRASTORNO }\end{array}$ & $\underset{(\mathbf{n})}{\text { MUESTRA }}$ & EDAD & INTERVENCIÓN & \multicolumn{3}{|c|}{$\begin{array}{l}\text { DISEÑO/CONDICIONES DE } \\
\text { INVESTIGACIÓN }\end{array}$} & \multirow{3}{*}{$\begin{array}{l}\text { MEDIDA DE } \\
\text { EVALUACIÓN } \\
\\
\text { VAS }\end{array}$} & \multirow[t]{2}{*}{ RESULTADOS } \\
\hline & & & & & Duración & Grupos & Medidas & & \\
\hline $\begin{array}{l}\text { Benta et al. } \\
\text { (2015) }\end{array}$ & $\begin{array}{l}\text { Trastorno por } \\
\text { ansiedad social }\end{array}$ & 5 & $\begin{array}{l}\text { Entre } \\
23-29\end{array}$ & $\begin{array}{l}\text { Speakeasy-a Mobile } \\
\text { Assistant }\end{array}$ & 1 semana & $\begin{array}{l}\text { No sigue } \\
\text { metodología de } \\
\text { ensayo clínico } \\
\text { randomizado }\end{array}$ & $\begin{array}{l}\text { Pre y Post } \\
\text { cuestionarios }\end{array}$ & & $\begin{array}{l}\text { Los revisores indicaron que la aplicación se ajusta al modelo cognitivo-conductual de la ansiedad social } \\
\text { y los ejercicios abordan los mecanismos psicológicos que se cree están involucrados en este problema de } \\
\text { salud mental. } \\
\text { El dispositivo móvil podría ser útil para la evaluación de la ansiedad en tiempo real y las emociones } \\
\text { asociadas. }\end{array}$ \\
\hline $\begin{array}{l}\text { Campos et al. } \\
\text { (2016) }\end{array}$ & Fobia a volar & 57 & 18 años & $\begin{array}{l}\text { Tratamiento basado } \\
\text { en Internet para la } \\
\text { fobia al vuelo }\end{array}$ & 3-4 semanas & $\begin{array}{l}\text { Metodología de } \\
\text { ensayo clínico } \\
\text { randomizado. } \\
\text { Grupo } 1 \\
\text { Grupo } 2 \\
\text { Grupo } 3\end{array}$ & $\begin{array}{l}\text { Pre y Post } \\
\text { Seguimiento } \\
3-12 \text { meses }\end{array}$ & $\begin{array}{l}\text { ADIS-IV } \\
\text { FFQ-II } \\
\text { FFS } \\
\text { Escalas miedo } \\
\text { evitación } \\
\text { Escala mejora } \\
\text { paciente }\end{array}$ & $\begin{array}{l}\text { El uso de un tratamiento basado en Internet podría tener ventajas notables para superar las limitaciones de } \\
\text { la exposición in vivo, de acceso al tratamiento, aceptación, cumplimiento y rentabilidad de la intervención. } \\
\text { Estas intervenciones autoaplicadas mejoran la posibilidad de llegar a las personas necesitadas. El uso de } \\
\text { las TIC puede tener una mejor aceptación entre los pacientes y los terapeutas porque producen niveles de } \\
\text { ansiedad más bajos promoviendo así una mejor adherencia y evitando los abandonos. }\end{array}$ \\
\hline $\begin{array}{l}\text { Ekberg et al. } \\
\text { (2011) }\end{array}$ & $\mathrm{TA}$ & 250 & $\begin{array}{l}\text { Entre } \\
18-25\end{array}$ & $\begin{array}{l}\text { TCC y Teléfono } \\
\text { móvil }\end{array}$ & $\begin{array}{l}\text { Periodo de } \\
3 \text { meses de } \\
6 \text { sesiones } \\
(45 \mathrm{~min})\end{array}$ & $\begin{array}{l}\text { No sigue } \\
\text { metodología de } \\
\text { ensayo clínico } \\
\text { randomizado. } \\
\text { CBT-ubicuo } \\
\text { CBT placebo } \\
\text { CBT- TAU }\end{array}$ & $\begin{array}{l}\text { PRE y POST } \\
\text { Seguimiento } \\
\text { a los } 6 \text { meses }\end{array}$ & $\begin{array}{l}\text { HADS- A } \\
\text { BAI } \\
\text { GHQ-12 }\end{array}$ & $\begin{array}{l}\text { No se encontraron diferencias significativas entre la psicoterapia cara a cara y la asistida por computadora } \\
\text { para la ansiedad y no hay diferencias entre el sistema utilizado. } \\
\text { Este protocolo para uso en la práctica clínica de primera línea, en el que se analizan la efectividad, la } \\
\text { adherencia y el papel de los terapeutas, proporcionará evidencia de lo que son tratamientos de TCC verda- } \\
\text { deramente valiosos respaldados por teléfono y una guía para la introducción más amplia de la TCC en los } \\
\text { servicios de salud. }\end{array}$ \\
\hline $\begin{array}{l}\text { Gary y } \\
\text { Amresh } \\
\text { (2017) }\end{array}$ & Ansiedad & $\begin{array}{l}177 \\
(132 \text { jóvenes } \\
\text { y } 45 \text { provee- } \\
\text { dores) }\end{array}$ & $\begin{array}{l}8-12 \text { años } \\
(9.65)\end{array}$ & "Reach App" & $\begin{array}{l}2 \text { semanas } \\
6 \text { sesiones } \\
(20-30 \mathrm{~min})\end{array}$ & $\begin{array}{l}\text { No sigue } \\
\text { metodología de } \\
\text { ensayo clínico } \\
\text { randomizado. }\end{array}$ & $\begin{array}{l}\text { Prevención e } \\
\text { intervención } \\
\text { temprana }\end{array}$ & $\begin{array}{l}\text { USE } \\
\text { RPS }\end{array}$ & $\begin{array}{l}\text { El puntaje general de uso de la app fue bueno ( }(\mathrm{M}=33.35 \text {; el rango posible es de } 0 \text { a } 40 \text { ) y las estimacio- } \\
\text { nes medias para las cinco dimensiones de uso fueron excelentes con un estigma bajo }(\mathrm{M}=2.41 \text {; el rango } \\
\text { posible es de } 0 \text { a 10). } \\
\text { La preocupación por el estigma para los jóvenes al usar la aplicación fue baja (M }=2.48 \text { de } 10, \mathrm{SD}=1.75) \text {, } \\
\text { sugiriendo alta aceptabilidad social. } \\
\text { Los resultados mostraron que la aplicación de prevención de la ansiedad y la intervención temprana } \\
\text { REACH fue altamente valorada positivamente por la mayoría de los jóvenes. Los mensajes desplegados } \\
\text { por la tecnología fueron calificados como útiles y claros; y la aplicación produjo una alta satisfacción y } \\
\text { aceptabilidad social. }\end{array}$ \\
\hline $\begin{array}{l}\text { Christensen et } \\
\text { al. (2014) }\end{array}$ & TAG & 558 & $\begin{array}{l}18-30 \\
\text { años }\end{array}$ & $\begin{array}{l}\text { Intervención web, } \\
\text { Ensayo iChill }\end{array}$ & $\begin{array}{l}5 \text { interven- } \\
\text { ciones en } 10 \\
\text { semanas }\end{array}$ & $\begin{array}{l}\begin{array}{l}\text { No sigue } \\
\text { metodología de } \\
\text { ensayo clínico } \\
\text { randomizado. }\end{array} \\
\text { grupo } 1 \\
\text { grupo 2 } \\
\text { grupo 3 } \\
\text { grupo } 4 \\
\text { grupo } 5\end{array}$ & $\begin{array}{l}\text { Pre y Post } \\
\text { Seguimiento } \\
6 \text { y } 12 \text { meses }\end{array}$ & $\begin{array}{l}\text { MINI } \\
\text { ASI } \\
\text { GAD -7 } \\
\text { PSWQ } \\
\text { CES-D } \\
\text { AUDIT } \\
\text { PHQ-9 }\end{array}$ & $\begin{array}{l}\text { Se encontraron tasas más altas de trabajo a tiempo completo entre los que recibieron el sitio web activo con } \\
\text { recordatorios por correo electrónico }(37.8 \%, 211 / 558) \text { y tasas más bajas entre los que recibieron el sitio web } \\
\text { con recordatorios telefónicos. } \\
\text { No hubo diferencias significativas entre el Grupo } 4 \text { (Control) y los Grupos } 1 \text { (Sitio web activo), } 2 \text { (Sitio } \\
\text { web activo con teléfono), } 3 \text { (Sitio web activo con correo electrónico), o } 5 \text { (Control con teléfono) en ningún } \\
\text { seguimiento. } \\
\text { No encontramos evidencia de que una serie de intervenciones activas breves se asociaran con mejores } \\
\text { resultados de ansiedad en el post-test o a los } 6 \text { o } 12 \text { meses. }\end{array}$ \\
\hline $\begin{array}{l}\text { Christoforou } \\
\text { et al. (2017) }\end{array}$ & Agorafobia & 140 & $>18$ años & $\begin{array}{l}\text { App “Agoraphobia } \\
\text { Free” }\end{array}$ & 12 semanas & $\begin{array}{l}\text { Metodología de } \\
\text { ensayo clínico } \\
\text { randomizado. } \\
\text { Grupo experi- } \\
\text { mental } \\
\text { Grupo control }\end{array}$ & Pre y Post & PAS & $\begin{array}{l}\text { Los puntajes de severidad de los síntomas disminuyeron en ambos grupos, pero no hubo evidencia de que } \\
\text { los cambios fueron significativamente mayores entre los participantes del grupo sin agorafobia que entre los } \\
\text { del grupo sin estrés. Tanto para la app Agorafobia libre }(\mathrm{n}=73 \text { ) como para Libre de estrés }(\mathrm{n}=69 \text { ), hubo } \\
\text { mejoras estadísticamente significativas en la gravedad de los síntomas desde el inicio hasta final. Hubo } \\
\text { reducciones significativas en la gravedad de los síntomas en ambos grupos a lo largo del tiempo, pero esas } \\
\text { reducciones fueron equivalentes en los } 2 \text { grupos. }\end{array}$ \\
\hline
\end{tabular}


Tabla 2. Descripción de los estudios seleccionados (continuación)

\begin{tabular}{|c|c|c|c|c|c|c|c|c|c|}
\hline ESTUDIO & $\begin{array}{c}\text { TIPO DE } \\
\text { TRASTORNO }\end{array}$ & $\underset{(\mathbf{n})}{\text { MUESTRA }}$ & EDAD & INTERVENCIÓN & \multicolumn{3}{|c|}{$\begin{array}{l}\text { DISEÑO/CONDICIONES DE } \\
\text { INVESTIGACIÓN }\end{array}$} & $\begin{array}{l}\text { MEDIDA DE } \\
\text { EVALUACIÓN }\end{array}$ & RESULTADOS \\
\hline & & & & & Duración & Grupos & Medidas & \multirow[b]{2}{*}{$\begin{array}{l}\text { STAI } \\
\text { PANAS } \\
\text { VAS } \\
\text { UCL-SUS } \\
\text { ITC-SOPI } \\
\text { STAI-Y }\end{array}$} & \multirow[b]{2}{*}{$\begin{array}{l}\text { La condición de vídeo mostró una disminución significativa del nivel de ansiedad en el tiempo. Los con- } \\
\text { tenidos de audio y vídeo son efectivos para reducir los niveles de ansiedad en una muestra de personas } \\
\text { que viajan diariamente. Los resultados mostraron una disminución significativa del nivel de ansiedad } \\
\text { en las condiciones de Nonar y MP3. En la condición de Vidnar, hay un aumento significativo del nivel } \\
\text { de relajación desde el principio hasta el final de la intervención. Se confirma la eficacia de la narrativa } \\
\text { móvil, para inducir estados emocionales positivos en los usuarios, mejorar su bienestar en situaciones de } \\
\text { estrés de la vida cotidiana y crear un sentido profundo de presencia en el entorno propuesto }\end{array}$} \\
\hline $\begin{array}{l}\text { Grassi et al. } \\
(2009)\end{array}$ & $\begin{array}{l}\text { Ansiedad y } \\
\text { estrés }\end{array}$ & 120 & Entre 20/25 & $\begin{array}{l}\text { Uso de narrativas a } \\
\text { través del móvil }\end{array}$ & $\begin{array}{l}4 \text { sesiones } \\
10 \text { minutos }\end{array}$ & 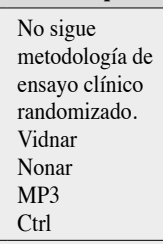 & Pre y Post & & \\
\hline $\begin{array}{l}\text { Hedman et al. } \\
\text { (2011) (1) }\end{array}$ & Fobia social & 126 & $\begin{array}{l}\text { ICBT }=35.2 \\
\text { CBTG }= \\
35.5\end{array}$ & $\begin{array}{l}\text { TCC basada en } \\
\text { Internet }\end{array}$ & $\begin{array}{l}\text { ICBT } 15 \\
\text { Semanas } \\
\text { (10 min por } \\
\text { semana) } \\
\text { CBGT }=14 \\
\text { sesiones } \\
\text { grupales en } \\
15 \text { semanas }\end{array}$ & $\begin{array}{l}\text { Metodología de } \\
\text { ensayo clínico } \\
\text { randomizado } \\
\text { ICBT }=64 \\
\text { CBGT }=62\end{array}$ & $\begin{array}{l}\text { Pre y Post } \\
\text { Seguimiento } \\
6 \text { meses }\end{array}$ & $\begin{array}{l}\text { MINI } \\
\text { MADRS-S } \\
\text { SPSQ } \\
\text { AUDIT } \\
\text { LSAS } \\
\text { SPS } \\
\text { BAI / ASI } \\
\text { QOLI } \\
\text { SCID-I/ SCID.II } \\
\text { GAF } \\
\text { CGI-1 }\end{array}$ & $\begin{array}{l}\text { En el postratamiento y el seguimiento de seis meses respectivamente, entre los grupos en la puntuación } \\
\text { LSAS fue de } 0,68 \text { a } 17,66 \text { y }-2,5 \text { a } 15,69 \text {, favoreciendo la ICBT. En el postratamiento, } 42 \text { participantes } \\
(66 \%) \text { en el grupo de ICBT se clasificaron como muy mejorados. Las pruebas de Wilcoxon mostraron } \\
\text { que los participantes que habían recibido ICBT mejoraron aún más en el seguimiento. Después del trata- } \\
\text { miento, } 18(31 \%) \text { participantes que habían recibido ICBT ya no cumplían con los criterios de diagnóstico } \\
\text { de ansiedad social. }\end{array}$ \\
\hline $\begin{array}{l}\text { Hedman et al. } \\
\text { (2013) (2) }\end{array}$ & Ansiedad & 81 & $\begin{array}{l}\text { ICBT }=39.3 \\
\text { CC }=38.8\end{array}$ & $\begin{array}{l}\text { TCC basada en } \\
\text { Internet }\end{array}$ & 12 semanas & $\begin{array}{l}\text { Metodología de } \\
\text { ensayo clínico } \\
\text { randomizado } \\
\text { ICBT }=40 \\
\text { CC }=41\end{array}$ & Pre y Post & $\begin{array}{l}\text { MINI } \\
\text { EAS }\end{array}$ & $\begin{array}{l}\text { Los participantes que recibieron TCC basada en Internet obtuvieron mejoras en los síntomas depresivos } \\
\text { y de ansiedad general. } \\
\text { Mejoras superiores en el grupo de TCC basado en Internet en comparación al grupo control. Hubo un } \\
\text { efecto de interacción significativo del tratamiento y el tiempo en todos los mediadores, menor riesgo per- } \\
\text { cibido y menos consecuencias negativas de la enfermedad, menor atención a las sensaciones corporales, } \\
\text { y una mayor intolerancia a la incertidumbre se asoció con niveles más bajos de ansiedad por la salud. }\end{array}$ \\
\hline $\begin{array}{l}\text { Lindner et al. } \\
\text { (2013) }\end{array}$ & $\begin{array}{l}\text { Ansiedad social } \\
\text { y trastorno de } \\
\text { pánico }\end{array}$ & 150 & $>18$ años & $\begin{array}{l}\text { TCC basada en inter- } \\
\text { net y aplicación de } \\
\text { teléfono inteligente }\end{array}$ & 24 semanas & $\begin{array}{l}\text { Metodología de } \\
\text { ensayo clínico } \\
\text { randomizado } \\
\text { iCBT (1) } \\
\text { iCBT ( } 2 \text { ) } \\
\text { lista espera }\end{array}$ & $\begin{array}{l}\text { Pre y Post } \\
\text { Seguimiento } \\
12,36 \text { meses }\end{array}$ & $\begin{array}{l}\text { (PHQ-9 } \\
\text { QOLI } \\
\text { PDSS-SR } \\
\text { LSAS-SR } \\
\text { GAD-7 }\end{array}$ & $\begin{array}{l}\text { Los grupos de tratamiento vieron reducción de la ansiedad general }(\mathrm{d}=0,39) \text { y social }(\mathrm{d}=0.70) \text {, pero } \\
\text { no los síntomas de pánico }(\mathrm{d}=0.05) \text { en comparación con el grupo de la lista de espera, sin embargo, } \\
\text { no se observaron diferencias en los resultados entre las intervenciones guiadas y no guiadas. La ACT } \\
\text { administrada por Internet es apropiada para tratar la ansiedad social. Las aplicaciones para teléfonos } \\
\text { inteligentes pueden compensar parcialmente la falta de apoyo del terapeuta. }\end{array}$ \\
\hline $\begin{array}{l}\text { Ly et al. } \\
\text { (2012) }\end{array}$ & $\begin{array}{l}\text { Ansiedad estrés, } \\
\text { y depresión, }\end{array}$ & 11 & $\begin{array}{l}>18 \text { años } \\
(29.5)\end{array}$ & $\begin{array}{l}\text { Aplicación de } \\
\text { teléfono inteligente } \\
\text { basada en la terapia } \\
\text { de aceptación y } \\
\text { compromiso. }\end{array}$ & 4 semanas & $\begin{array}{l}\text { No sigue } \\
\text { metodología de } \\
\text { ensayo clínico } \\
\text { randomizado }\end{array}$ & Pre y Post & $\begin{array}{l}\text { BEVS, AAQ-II, } \\
\text { SWLS y DASS- } 21\end{array}$ & $\begin{array}{l}\text { Las pruebas mostraron diferencias significativas entre la intervención previa y posterior. No se encontra- } \\
\text { ron diferencias significativas en satisfacción con la vida global o en síntomas depresivos y ansiosos. En } \\
\text { general, los participantes informaron que la intervención les hizo pensar más en sus valores y comporta- } \\
\text { mientos. La mayoría de los participantes usaron la aplicación un par de veces por semana y algunos de } \\
\text { los participantes la usaron cuando estaban sentados en el autobús, el metro, el tren, etc. }\end{array}$ \\
\hline $\begin{array}{l}\text { Mohr et al. } \\
\text { (2017) }\end{array}$ & $\begin{array}{l}\text { TAG y Depre- } \\
\text { sión }\end{array}$ & 96 & 18 & $\begin{array}{l}\text { IntelliCare: un con- } \\
\text { junto de aplicaciones }\end{array}$ & 8 semanas & $\begin{array}{l}\text { No sigue } \\
\text { metodología de } \\
\text { ensayo clínico } \\
\text { randomizado. }\end{array}$ & $\begin{array}{l}\text { Pre y Post } \\
\text { Seguimiento }\end{array}$ & $\begin{array}{l}\text { PHQ -9 } \\
\text { GAD -7 } \\
\text { MINI }\end{array}$ & 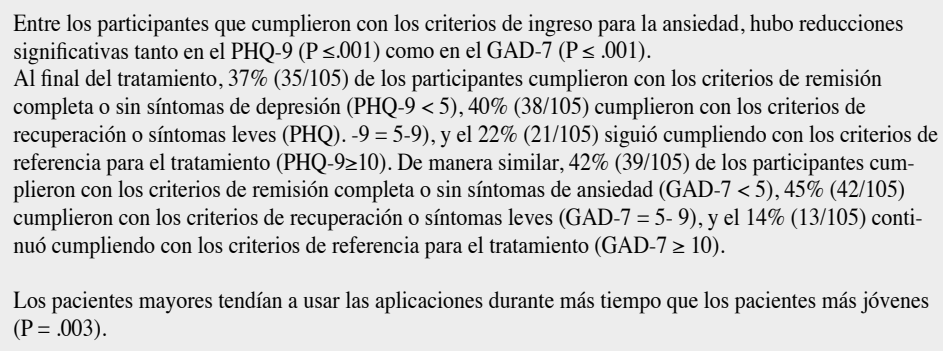 \\
\hline
\end{tabular}


Tabla 2. Descripción de los estudios seleccionados (continuación)

\begin{tabular}{|c|c|c|c|c|c|c|c|c|c|}
\hline ESTUDIO & $\begin{array}{c}\text { TIPO DE } \\
\text { TRASTORNO }\end{array}$ & $\underset{\text { (n) }}{\text { MUESTRA }}$ & EDAD & INTERVENCIÓN & \multicolumn{3}{|c|}{$\begin{array}{l}\text { DISEÑO/CONDICIONES DE } \\
\text { INVESTIGACIÓN }\end{array}$} & $\begin{array}{l}\text { MEDIDA DE } \\
\text { EVALUACIÓN }\end{array}$ & RESULTADOS \\
\hline & & & & & Duración & Grupos & Medidas & \multirow[b]{2}{*}{$\begin{array}{l}\text { GAD } 7 \\
\text { PSWQ } \\
\text { BAI } \\
\text { STAI-Y } \\
\text { HAM -A } \\
\text { VAS - A }\end{array}$} & \multirow[b]{2}{*}{$\begin{array}{l}\text { Hubo disminución significativa en GAD - 7/BAI/STAI - y en el grupo VRMB y en PSWQ en VRM. En } \\
\text { cuestionarios pre y post tratamiento no hubo diferencias significativas (solo destacan con p <.1 GAD-7 } \\
\text { y STAI-Y2) Con la RV ambos grupos mejoraron al final del tratamiento. El 91\% de los participantes } \\
\text { apoyaron el uso del móvil (PDA). El uso de la RV con Biofeedback aumenta el efecto de la RV. }\end{array}$} \\
\hline $\begin{array}{l}\text { Pallavicini et } \\
\text { al. (2009) }\end{array}$ & TAG & 12 & Entre $18-50$ & $\begin{array}{l}\text { BiofeedBack, Reali- } \\
\text { dad Virtual y teléfono } \\
\text { móvil }\end{array}$ & $\begin{array}{l}\text { Periodo } \\
\text { quincenal } \\
\text { de } 8 \text { sesio- } \\
\text { nes. } \\
\text { Proyecto } \\
\text { INTREPID }\end{array}$ & $\begin{array}{l}\text { Metodología de } \\
\text { ensayo clínico } \\
\text { randomizado } \\
\text { LE: } 4 \\
\text { VRM:4 } \\
\text { VRMB:4 }\end{array}$ & PRE y POST & & \\
\hline $\begin{array}{l}\text { Per Carlbring } \\
\text { et al. (2007) }\end{array}$ & Fobia social & 57 & $\begin{array}{l}\text { Tratamiento } \\
=32.4 \\
\text { Grupo con- } \\
\text { trol }=32.9\end{array}$ & $\begin{array}{l}\text { TCC con soporte de } \\
\text { teléfono }\end{array}$ & 9 semanas & $\begin{array}{l}\text { Grupo LE } \\
\text { Grupo tratamiento }\end{array}$ & $\begin{array}{l}\text { Metodología } \\
\text { de ensayo } \\
\text { clínico ran- } \\
\text { domizado } \\
\text { Pre y Post } \\
\text { Seguimiento } \\
1 \text { años }\end{array}$ & $\begin{array}{l}\text { SPSQ } \\
\text { MADRS - } \\
\text { SCID } \\
\text { LSAS-SR } \\
\text { SPS } \\
\text { SIAS } \\
\text { SPSQ } \\
\text { BAI } \\
\text { QoLI }\end{array}$ & $\begin{array}{l}\text { El grupo de intervención había mejorado significativamente entre las evaluaciones previas y posteriores } \\
\text { al tratamiento (t281466.3-2.2, P50.001), mientras que el grupo control no. Además, después del tratamien- } \\
\text { to, el grupo tratado tuvo niveles de ansiedad social más bajos en todas las escalas en comparación con } \\
\text { los controles. Los valores de p indicaron que el grupo de intervención había mejorado significativamente } \\
\text { entre el tratamiento previo y la evaluación posterior al tratamiento. El grupo de intervención tuvo niveles } \\
\text { de angustia más bajos. Específicamente, los participantes tratados lograron mejoras significativas en } \\
\text { medidas de ansiedad social, miedo, evitación, depresión y ansiedad general. }\end{array}$ \\
\hline $\begin{array}{l}\text { Pham et al. } \\
\text { (2016) }\end{array}$ & $\begin{array}{l}\text { Ansiedad } \\
\text { Pánico }\end{array}$ & 63 & 18 años & $\begin{array}{l}\text { mHealth Game: } \\
\text { "FLOWY" }\end{array}$ & 4 semanas & $\begin{array}{l}\text { Grupo interven- } \\
\text { ción } \\
\text { Grupo control }\end{array}$ & $\begin{array}{l}\text { Metodología } \\
\text { de ensayo } \\
\text { clínico ran- } \\
\text { domizado } \\
\text { Pre y Post }\end{array}$ & $\begin{array}{l}\text { GAD-7, PDSS-SR, } \\
\text { Nijmegen QLES- } \\
\text { Q-SF } \\
\text { ASI-3 } \\
\text { OASIS } \\
\text { PDSS-SR } \\
\text { eHEALS }\end{array}$ & $\begin{array}{l}\text { El } 84 \% \text { de los participantes en el grupo de intervención jugaron de forma proactiva a ‘Flowy al menos } \\
\text { una vez durante un mínimo de } 1 \text { minuto durante el estudio. } \\
\text { Al concluir el estudio, los participantes de ambos grupos tuvieron puntuaciones mejoradas en ansiedad, } \\
\text { pánico, hiperventilación y calidad de vida. Mostraron un aumento en la calidad de vida (Dmean }=4.570 \text {; } \\
\text { F1,61 = 4.845, } \mathrm{P}=0.034 \text { ) y una reducción en la ansiedad (Dmean }=0,911 ; \mathrm{F} 1,61=1,049, \mathrm{P}=0,310 \text { ), } \\
\text { pánico (Dmean }=0,108 ; \mathrm{F} 1,61=0,012, \mathrm{P}=0,914 \text { ) e hiperventilación (Dmean }=0.523 ; \mathrm{F} 1,61=0.055, \mathrm{P} \\
=0.816 \text { ) en el grupo de intervención jugando Flowy. En particular, las puntuaciones de hiperventilación } \\
\text { del grupo de intervención disminuyeron significativamente. }\end{array}$ \\
\hline $\begin{array}{l}\text { Ly et al. } \\
\text { (2012) }\end{array}$ & $\begin{array}{l}\text { Ansiedad estrés, } \\
\text { y depresión, }\end{array}$ & 11 & $\begin{array}{l}>18 \text { años } \\
(29.5)\end{array}$ & $\begin{array}{l}\text { Aplicación de } \\
\text { teléfono inteligente } \\
\text { basada en la terapia } \\
\text { de aceptación y } \\
\text { compromiso. }\end{array}$ & 4 semanas & $\begin{array}{l}\text { No sigue } \\
\text { metodología de } \\
\text { ensayo clínico } \\
\text { randomizado }\end{array}$ & Pre y Post & $\begin{array}{l}\text { BEVS, AAQ-II, } \\
\text { SWLS y DASS-21 }\end{array}$ & $\begin{array}{l}\text { Las pruebas mostraron diferencias significativas entre la intervención previa y posterior. No se encontra- } \\
\text { ron diferencias significativas en satisfacción con la vida global o en síntomas depresivos y ansiosos. En } \\
\text { general, los participantes informaron que la intervención les hizo pensar más en sus valores y comporta- } \\
\text { mientos. La mayoría de los participantes usaron la aplicación un par de veces por semana y algunos de } \\
\text { los participantes la usaron cuando estaban sentados en el autobús, el metro, el tren, etc. }\end{array}$ \\
\hline $\begin{array}{l}\text { Per Carlbring } \\
\text { et al. (2007) }\end{array}$ & Fobia social & 57 & $\begin{array}{l}\text { Tratamiento } \\
=32.4 \\
\text { Grupo con- } \\
\text { trol }=32.9\end{array}$ & $\begin{array}{l}\text { TCC con soporte de } \\
\text { teléfono }\end{array}$ & 9 semanas & $\begin{array}{l}\text { Grupo LE } \\
\text { Grupo tratamiento }\end{array}$ & $\begin{array}{l}\text { Metodología } \\
\text { de ensayo } \\
\text { clínico ran- } \\
\text { domizado } \\
\text { Pre y Post } \\
\text { Seguimiento } \\
1 \text { años }\end{array}$ & $\begin{array}{l}\text { SPSQ } \\
\text { MADRS - S } \\
\text { SCID } \\
\text { LSAS-SR } \\
\text { SPS } \\
\text { SIAS } \\
\text { SPSQ } \\
\text { BAI } \\
\text { QoLI }\end{array}$ & $\begin{array}{l}\text { El grupo de intervención había mejorado significativamente entre las evaluaciones previas y posteriores } \\
\text { al tratamiento (t281/46.3-2.2, P50.001), mientras que el grupo control no. Además, después del tratamien- } \\
\text { to, el grupo tratado tuvo niveles de ansiedad social más bajos en todas las escalas en comparación con } \\
\text { los controles. Los valores de p indicaron que el grupo de intervención había mejorado significativamente } \\
\text { entre el tratamiento previo y la evaluación posterior al tratamiento. El grupo de intervención tuvo niveles } \\
\text { de angustia más bajos. Específicamente, los participantes tratados lograron mejoras significativas en } \\
\text { medidas de ansiedad social, miedo, evitación, depresión y ansiedad general. }\end{array}$ \\
\hline
\end{tabular}

\begin{tabular}{|c|c|c|c|c|c|c|c|}
\hline $\begin{array}{l}\text { Pramana et al. } \\
\text { (2014) (1) }\end{array}$ & Ansiedad & 9 & $\begin{array}{l}11.33 \\
\text { (9-14 años) }\end{array}$ & $\begin{array}{l}\text { SmartCAT: platafor- } \\
\text { ma mHealth }\end{array}$ & $\begin{array}{l}\mathrm{CBT}=16 \\
\text { sesiones } \\
\mathrm{BCBT}=8 \\
\text { sesiones }\end{array}$ & $\begin{array}{l}\text { No sigue } \\
\text { metodología de } \\
\text { ensayo clínico } \\
\text { randomizado. } \\
\text { CBT=3 } \\
\text { BCBT }=6\end{array}$ & $\begin{array}{l}\text { EAT-R } \\
\text { ESC } \\
\text { USC }\end{array}$ \\
\hline
\end{tabular}

Los resultados sugieren que una aplicación SmartCAT puede integrarse con éxito en la TCC para niños con trastornos de ansiedad. Los pacientes estaban usando activamente la aplicación, con un promedio de 5.36 entradas por sesión, y cumplían con el protocolo de tratamiento. La inclusión del establecimiento de objetivos a través de recompensas puede potencialmente aumentar la participación de los pacientes en el tratamiento a través de la práctica de entrenamiento de habilidades.

Los resultados indicaron reducciones similares en los síntomas de ansiedad para BCBT + EMI en comparación con la TCC estándar después del tratamiento. 
Tabla 2. Descripción de los estudios seleccionados (continuación)

\begin{tabular}{|c|c|c|c|c|c|c|c|c|c|}
\hline ESTUDIO & $\begin{array}{l}\text { TIPO DE } \\
\text { TRASTORNO }\end{array}$ & $\begin{array}{l}\text { MUESTRA } \\
\text { (n) }\end{array}$ & EDAD & INTERVENCIÓN & \multicolumn{3}{|c|}{$\begin{array}{l}\text { DISEÑO/CONDICIONES DE } \\
\text { INVESTIGACIÓN }\end{array}$} & $\begin{array}{l}\text { MEDIDA DE } \\
\text { EVALUACIÓN }\end{array}$ & RESULTADOS \\
\hline & & & & & Duración & Grupos & Medidas & \multirow[b]{2}{*}{$\begin{array}{l}\text { GAD-7, PDSS-SR, } \\
\text { Nijmegen QLES- } \\
\text { Q-SF } \\
\text { ASI-3 } \\
\text { OASIS } \\
\text { PDSS-SR } \\
\text { eHEALS }\end{array}$} & \multirow[b]{2}{*}{$\begin{array}{l}\text { El } 84 \% \text { de los participantes en el grupo de intervención jugaron de forma proactiva a 'Flowy al menos } \\
\text { una vez durante un mínimo de } 1 \text { minuto durante el estudio. } \\
\text { Al concluir el estudio, los participantes de ambos grupos tuvieron puntuaciones mejoradas en ansiedad, } \\
\text { pánico, hiperventilación y calidad de vida. Mostraron un aumento en la calidad de vida (Dmean }=4.570 \text {; } \\
\mathrm{F} 1,61=4.845, \mathrm{P}=0.034 \text { ) y una reducción en la ansiedad (Dmean }=0,911 ; \mathrm{F} 1,61=1,049, \mathrm{P}=0,310 \text { ), } \\
\text { pánico (Dmean }=0,108 ; \mathrm{F} 1,61=0,012, \mathrm{P}=0,914 \text { ) e hiperventilación (Dmean }=0.523 ; \mathrm{F} 1,61=0.055, \mathrm{P} \\
=0.816 \text { ) en el grupo de intervención jugando Flowy. En particular, las puntuaciones de hiperventilación } \\
\text { del grupo de intervención disminuyeron significativamente. }\end{array}$} \\
\hline $\begin{array}{l}\text { Pham et al. } \\
\text { (2016) }\end{array}$ & $\begin{array}{l}\text { Ansiedad } \\
\text { Pánico }\end{array}$ & 63 & 18 años & $\begin{array}{l}\text { mHealth Game: } \\
\text { "FLOWY" }\end{array}$ & 4 semanas & $\begin{array}{l}\text { Grupo interven- } \\
\text { ción } \\
\text { Grupo control }\end{array}$ & $\begin{array}{l}\text { Metodología } \\
\text { de ensayo } \\
\text { clínico ran- } \\
\text { domizado } \\
\text { Pre y Post }\end{array}$ & & \\
\hline $\begin{array}{l}\text { Pramana et al. } \\
\text { (2018) (2) }\end{array}$ & $\begin{array}{l}\text { TAG } \\
\text { TA Social } \\
\text { TA Separación }\end{array}$ & 35 & $\begin{array}{l}11.19 \\
(9-14)\end{array}$ & $\begin{array}{l}\text { TCC y APP móvil } \\
\text { Proyecto SmarCAT }\end{array}$ & 8 sesiones & $\begin{array}{l}\text { No sigue } \\
\text { metodología de } \\
\text { ensayo clínico } \\
\text { randomizado. } \\
\text { Fase de prueba } \\
\text { beta:5 } \\
\text { Fase de prueba } \\
\text { abierta: } 30\end{array}$ & $\begin{array}{l}\text { PRE y POST } \\
\text { Seguimiento } \\
\text { semanal }\end{array}$ & (K-SADS-PL) & $\begin{array}{l}\text { Los participantes estaban más motivados y tenían más probabilidades de participar en el aprendizaje de } \\
\text { habilidades de TCC utilizando un entorno de aprendizaje interactivo y divertido, como los juegos. } \\
\text { Los participantes se mostraron cómodos al utilizar la aplicación en parte de su rutina diaria. Declararon } \\
\text { que la aplicación era fácil de usar y la encontraron útil cuando experimentaban ansiedad. } \\
\text { El resultado de la implementación indica que el sistema SmartCAT se ha utilizado como se esperaba y } \\
\text { sugiere que la inclusión de la gamificación puede aumentar efectivamente la participación y retención } \\
\text { del usuario. }\end{array}$ \\
\hline $\begin{array}{l}\text { Proudfoot et } \\
\text { al. (2013) }\end{array}$ & $\begin{array}{l}\text { Ansiedad, } \\
\text { estrés y } \\
\text { depresión }\end{array}$ & 720 & 38.9 & $\begin{array}{l}\text { Teléfono móvil y } \\
\text { programa a través } \\
\text { de la web. Proyecto } \\
\text { MyCompass }\end{array}$ & $\begin{array}{l}3 \text { sesiones } \\
\text { por } 12 \\
\text { módulos }\end{array}$ & $\begin{array}{l}\text { Metodología de } \\
\text { ensayo clínico } \\
\text { randomizado } \\
\text { MC=242 } \\
\text { AC=248 } \\
\text { LE }=230\end{array}$ & $\begin{array}{l}\text { Pre y Post } \\
\text { Seguimiento } \\
12 \text { semanas } \\
\text { (MC y AC) } \\
\text { seguimiento } \\
19 \text { semanas } \\
\text { (LE) }\end{array}$ & $\begin{array}{l}\text { DASS-21 } \\
\text { WSAS }\end{array}$ & $\begin{array}{l}\text { Mejoría significativamente mayor en la postintervención para el grupo myCompass en comparación con } \\
\text { los grupos de AC y LE en las escalas de Depresión, Ansiedad, DASS Total y WSAS. } \\
\text { El } 83 \% \text { de los participantes de myCompass informaron que recomendarían el programa a otros, y el } \\
87.4 \% \text { indicó que volverían a usar el programa con gusto. } \\
\text { MyCompass mostró síntomas de depresión, ansiedad y estrés significativamente reducidos, y mejoró } \\
\text { significativamente los niveles de trabajo y funcionamiento social. } \\
\text { Las puntuaciones reducidas se mantuvieron a los } 3 \text { meses de seguimiento. }\end{array}$ \\
\hline $\begin{array}{l}\text { Spence et al. } \\
\text { (2011) }\end{array}$ & $\begin{array}{l}\text { TAG } \\
\text { Ansiedad por } \\
\text { separación } \\
\text { Fobia especifica }\end{array}$ & 115 & $\begin{array}{l}13.98 \\
(12 \text { y } 18 \\
\text { años) }\end{array}$ & $\begin{array}{l}\text { TCC basada en } \\
\text { internet } \\
\text { "BRAVE" }\end{array}$ & $\begin{array}{l}12 \text { semanas } \\
10 \text { sesiones } \\
60 \text { minutos }\end{array}$ & $\begin{array}{l}\text { Metodología de } \\
\text { ensayo clínico } \\
\text { randomizado } \\
\text { CLIN } \\
\text { NET } \\
\text { WLC }\end{array}$ & $\begin{array}{l}\text { Pre y Post } \\
6,12 \text { meses } \\
\text { seguimiento }\end{array}$ & $\begin{array}{l}\text { ADIS - C / P } \\
\text { CGAS } \\
\text { SCAS -C /P } \\
\text { CBCL } \\
\text { YSR }\end{array}$ & $\begin{array}{l}\text { Los resultados del estudio demuestran los beneficios significativos de entrega en línea de TCC para el tra- } \\
\text { tamiento de trastornos de ansiedad en adolescentes. Además, el programa en línea fue considerado como } \\
\text { un enfoque altamente creíble por parte de padres y adolescentes, en un nivel equivalente al de la TCC } \\
\text { basada en la clínica, y produjo calificaciones de satisfacción con el tratamiento de moderadas a altas. } \\
\text { Tanto Internet como la TCC basada en la clínica produjeron reducciones significativamente mayores en } \\
\text { la ansiedad y mayores mejoras en el funcionamiento general que el grupo de lista de espera en el punto } \\
\text { de evaluación de } 12 \text { semanas, sin diferencias significativas entre los formatos de tratamiento. }\end{array}$ \\
\hline $\begin{array}{l}\text { Villani et al. } \\
\text { (2013) }\end{array}$ & Ansiedad Estrés & 30 & 43 & Teléfono móvil & 4 semanas & $\begin{array}{l}\text { No sigue metodo- } \\
\text { logía de ensayo- } \\
\text { clínico randomiza- } \\
\text { do CE }=15 \\
\text { CC }=15\end{array}$ & Pre y Post & $\begin{array}{l}\text { MSP } \\
\text { STAI } \\
\text { COPE } \\
\text { JCQ }\end{array}$ & $\begin{array}{l}\text { Diferencias significativas entre CE y las condiciones del grupo de control relacionadas con la reducción } \\
\text { del estado de ansiedad. } \\
\text { Los cambios logrados dentro de cada sesión demostraron una disminución significativa y continua del } \\
\text { estado de ansiedad al final de cada sesión para el grupo experimental, pero no para el control. También se } \\
\text { identificó una disminución significativa del rasgo de ansiedad. }\end{array}$ \\
\hline $\begin{array}{l}\text { Wozney et al. } \\
\text { (2015) }\end{array}$ & TA & 17 & Entre 15-24 & $\begin{array}{l}\text { Programa Breathe: } \\
\text { TCC basado en } \\
\text { Internet }\end{array}$ & & $\begin{array}{l}\text { No sigue metodo- } \\
\text { logía de ensayo } \\
\text { clínico randomiza- } \\
\text { doCl=9 } \\
\mathrm{C} 2=8\end{array}$ & Post & $\begin{array}{l}\text { SUS } \\
\text { SUPR-Q } \\
\text { Entrevista semiestruc- } \\
\text { turada }\end{array}$ & $\begin{array}{l}\text { Las respuestas al contenido del programa y al cuestionario de usabilidad fueron positivas en todos los } \\
\text { ciclos de prueba y grupos de usuarios. } \\
\text { Los resultados sugieren que el compromiso con los jóvenes durante las fases tempranas del desarrollo de } \\
\text { la intervención es fundamental para adaptar las intervenciones terapéuticas en línea a lo que los jóvenes } \\
\text { piensan que funciona y por qué. } \\
\text { Diferencias en la forma en que los clínicos y los jóvenes pueden abordar las intervenciones basadas en } \\
\text { Internet. Los clínicos se centraron más en los aspectos legales y los mecanismos de gestión de riesgos } \\
\text { en un entorno en línea, mientras que los jóvenes se centraron más en la transparencia y la autonomía } \\
\text { personal. }\end{array}$ \\
\hline
\end{tabular}


Tabla 2. Descripción de los estudios seleccionados (continuación)

\begin{tabular}{|c|c|c|c|c|c|c|c|c|c|}
\hline ESTUDIO & $\begin{array}{c}\text { TIPO DE } \\
\text { TRASTORNO }\end{array}$ & $\underset{(\mathbf{n})}{\operatorname{MUESTRA}}$ & EDAD & INTERVENCIÓN & \multicolumn{3}{|c|}{$\begin{array}{l}\text { DISEÑO/CONDICIONES DE } \\
\text { INVESTIGACIÓN }\end{array}$} & $\begin{array}{l}\text { MEDIDA DE } \\
\text { EVALUACIÓN }\end{array}$ & RESULTADOS \\
\hline & & & & & Duración & Grupos & Medidas & \multirow[b]{2}{*}{$\begin{array}{l}\text { SPS } \\
\text { SIAS } \\
\text { SCID-I } \\
\text { BDI-II } \\
\text { IIP } \\
\text { SF-12 } \\
\text { BSI }\end{array}$} & \multirow[b]{2}{*}{$\begin{array}{l}\text { Ambos tratamientos activos superaron la LE en el post, } \mathrm{t}(119.46)=5.08, \mathrm{p}<.01, \mathrm{~d}=1.07 \text {, sin una dife- } \\
\text { rencia significativa entre las condiciones activas (app y PC) pero una tendencia a favor de la condición } \\
\text { de la aplicación, } \mathrm{t}(120.75)=1.71, \mathrm{p}=.09, \mathrm{~d}=0.30 \text {. El tratamiento móvil es al menos no menos eficaz } \\
\text { que la versión para PC. Las medidas compuestas de ansiedad social fueron } \mathrm{d}=0.07 \text { para App vs. PC (a } \\
\text { favor de App), } \mathrm{d}=0.89 \text { para App v. LE, y } \mathrm{d}=0.74 \text { para PC } \mathrm{v} \text {. LE. Los análisis de contraste indican que } \\
\text { las puntuaciones de seguimiento mejoraron con respecto al inicio. Ambos formatos de tratamiento fueron } \\
\text { efectivos para reducir los síntomas de ansiedad social y aumentar el bienestar psicológico después de } 12 \\
\text { semanas de tratamiento. }\end{array}$} \\
\hline $\begin{array}{l}\text { Stolz et al. } \\
\text { (2018) }\end{array}$ & Fobia social & 150 & $>18$ & Mobile app & 12 semanas & $\begin{array}{l}\text { Metodología de } \\
\text { ensayo clínico } \\
\text { randomizado } \\
\text { App } \\
\text { PC } \\
\text { LE }\end{array}$ & $\begin{array}{l}\text { Pre y Post } \\
\text { Seguimiento } \\
3 \text { meses }\end{array}$ & & \\
\hline
\end{tabular}

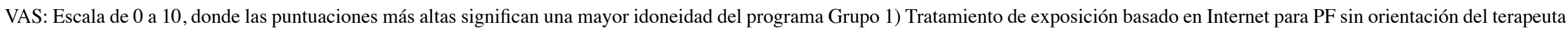

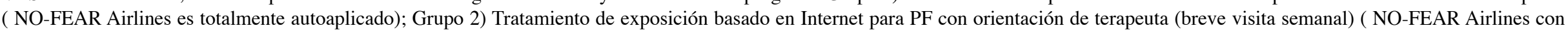

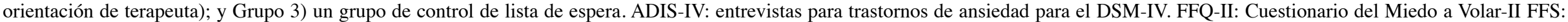

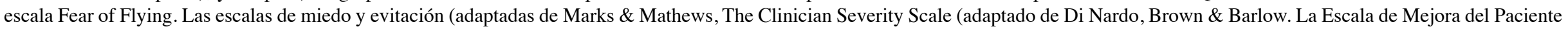
(Adaptada de la Escala de Impresión Global Clínica, CGI; Guy)

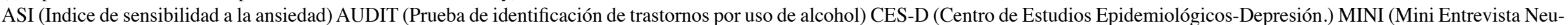

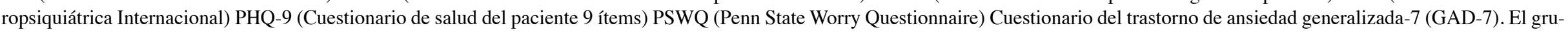

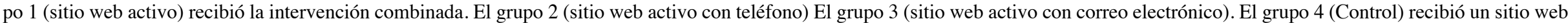

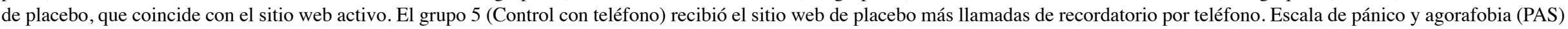

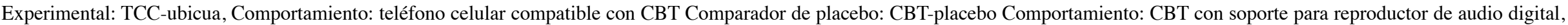

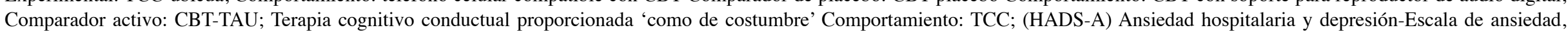

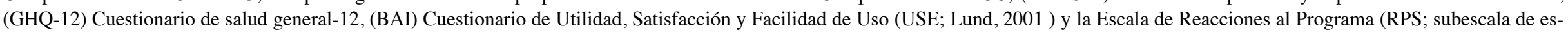
tigma; Rapee et al., 2006 ).

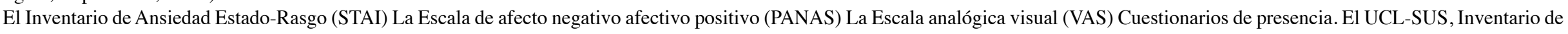

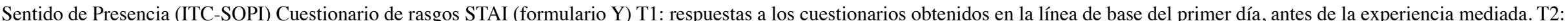

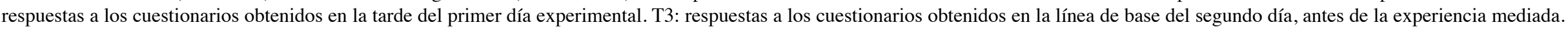

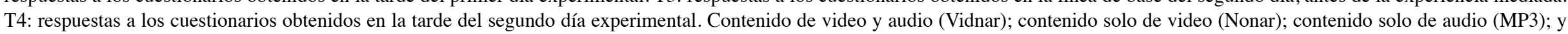

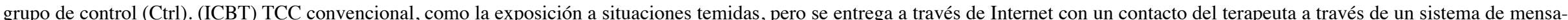

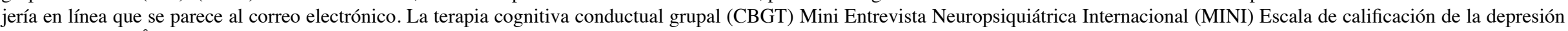

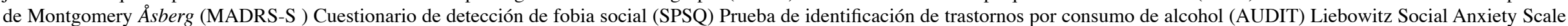

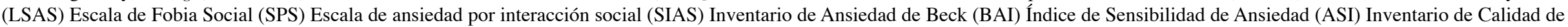
Vida (QOLI) ,SCID-II. Evaluación global de la Escala de funcionamiento (GAF) Escala de mejora de la impresión clínica global (CGI-I)

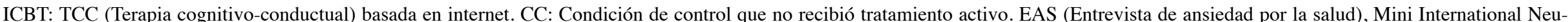

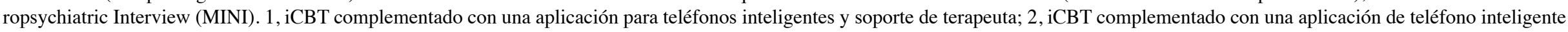

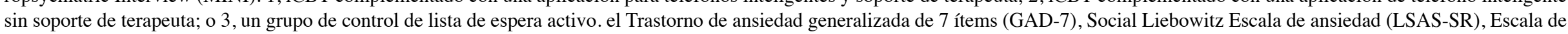

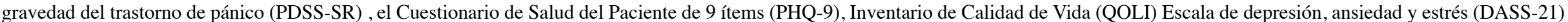
Encuesta del valor del ojo de buey (BEVS) Cuestionario de Aceptación y Acción II (AAQ-II) Escala de satisfacción con la vida (SWLS)

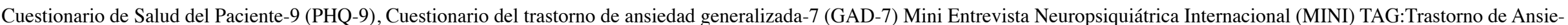

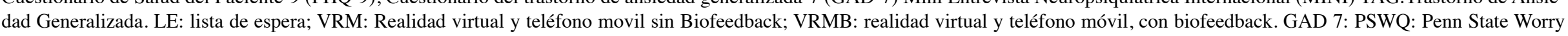

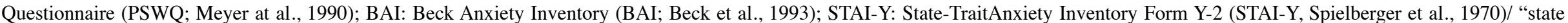
anxiety" (STAY-Y1)/ "trait anxiety" (STAY-Y2); HAM-A: Hamilton Anxiety Rating Scale (HAM-A; Hamilton, 1959); VAS - A: Visual Analogue Scale for Anxiety 


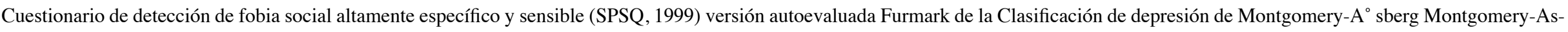

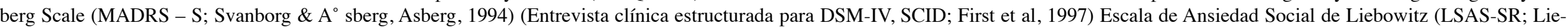

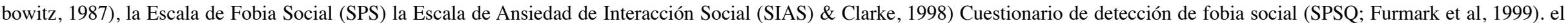

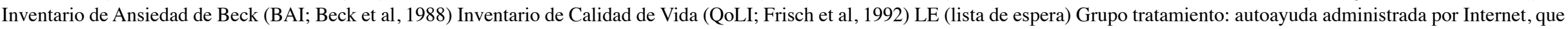

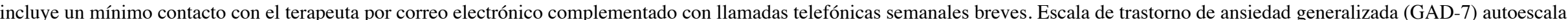

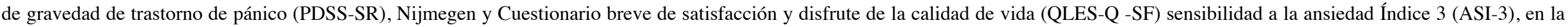
Escala de gravedad e insuficiencia de ansiedad general (OASIS). PDSS-SR: Panic Disorder Severity Scale. eHealth Literacy Scale (eHEALS)

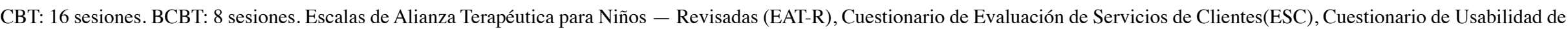

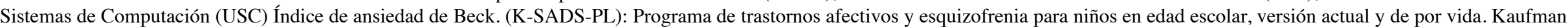

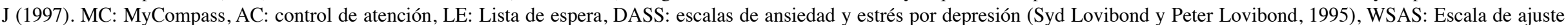
laboral y social

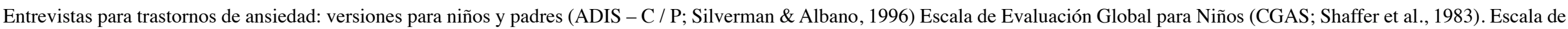

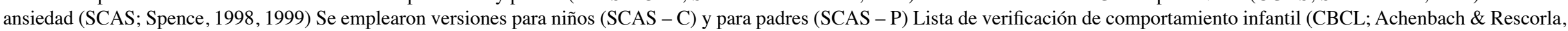

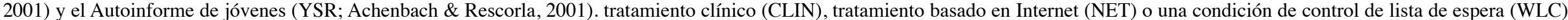

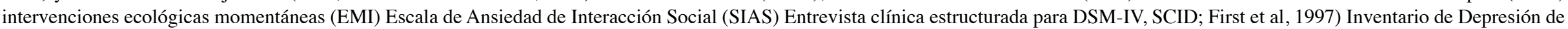

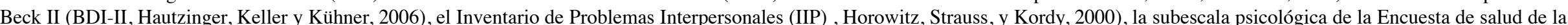

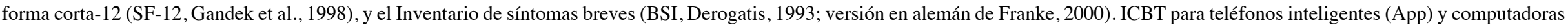
convencionales (PC) con un grupo de control de lista de espera (LE).

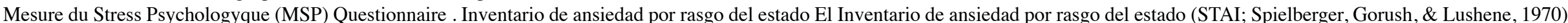

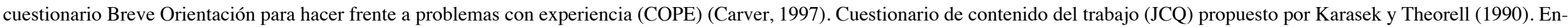

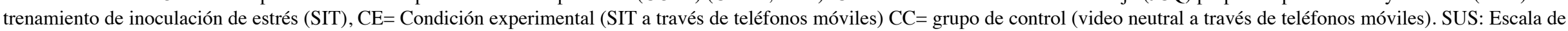
usabilidad del sistema (Bangor A, Kortum P, Miller J, 2009), SUPR-Q: Cuestionario de rango de percentil de experiencia de usuario estandarizado (Sauro J, Lewis J, 2012). 
Tabla 3. Descripción de los programas de intervención

\begin{tabular}{|c|c|c|}
\hline INTERVENCIÓN & AUTORES & DESCRIPCIÓN DEL PROGRAMA \\
\hline Speakeasy-a Mobile Assistant & Benta et al. (2015) & $\begin{array}{l}\text { La aplicación Speakeasy se enfoca en abordar la ansiedad social en situaciones de habla pública. SpeakEasy está destinado a ser un asistente móvil de autoayuda. } \\
\text { SpeakEasy es similar a otras aplicaciones, analiza las emociones y los pensamientos del usuario para extraer patrones de comportamiento, pero también está diseñado } \\
\text { para aumentar la autoconciencia y dominar la ansiedad social de una manera de autoayuda. }\end{array}$ \\
\hline $\begin{array}{l}\text { Tratamiento basado en Internet para } \\
\text { la fobia al vuelo }\end{array}$ & Campos et al. (2016) & $\begin{array}{l}\text { NO-FEAR Airlines Este programa permite a las personas que temen volar estar expuestas a imágenes y sonidos relacionados con sus miedos fóbicos en un ordenador } \\
\text { personal estándar desde su hogar. El protocolo de evaluación proporciona una evaluación corta con } 19 \text { preguntas sobre la fobia a volar. Dispone de un protocolo de } \\
\text { tratamiento que consta de } 3 \text { componentes terapéuticos: psicoeducación, exposición y sobre aprendizaje. La exposición se realiza a través de } 6 \text { escenarios compuestos de } \\
\text { imágenes y sonidos reales relacionados con el proceso de vuelo. }\end{array}$ \\
\hline Intervención web, Ensayo iChill & Christensen et al. (2014) & $\begin{array}{l}\text { Programa "e-couch", consistió en un programa integrado de psicoeducación (semanas 1-2), TCC (semanas 3-7), relajación (semanas 8-9) y promoción de la actividad } \\
\text { física (semana 10). }\end{array}$ \\
\hline App “Agoraphobia Free" & Christoforou et al. (2017) & $\begin{array}{l}\text { APP Agoraphobia Free (versión 0.8). La aplicación fue una intervención interactiva basada en juegos, con personajes tridimensionales y situaciones que simulan entor- } \\
\text { nos de la vida real. Específicamente, la aplicación presenta un ejemplo de caso de un personaje virtual que tenía agorafobia. El usuario debe guiarla, a través de la ayuda } \\
\text { del terapeuta virtual, para completar las diferentes tareas terapéuticas. }\end{array}$ \\
\hline TCC y Teléfono móvil & Ekberg et al. (2011) & Experimental: TCC-ubicua. Comportamiento: teléfono móvil compatible con CBT. Terapia cognitiva conductual con soporte de aplicaciones de teléfono móvil. \\
\hline "Reach App" & Gary y Amresh (2017) & En la pantalla de inicio de la aplicación $\boldsymbol{R E A C H}$, la página de inicio muestra cinco actividades (Relajación, Diario, PARADA, STICs, y Worryheads). \\
\hline Uso de narrativas a través del móvil & Grassi et al. (2009) & $\begin{array}{l}\text { La narrativa móvil se utilizó en el protocolo de este estudio, cuyo objetivo era verificar si una experiencia narrativa, asociada con el contenido de video, puede influir en } \\
\text { el estado emocional de los participantes. Fueron reclutados en el tren local (viaje de } 70 \text { minutos). Los pasajeros se dividieron en dos grupos según diferentes horarios. } \\
\text { Quienes participaron en el experimento fueron a Milán solo para asistir a una clase universitaria, no para un examen. Para apoyar la capacitación, desarrollaron conteni- } \\
\text { dos de audio y vídeo: el contenido de audio consistió en una narrativa. Voz que guio a los participantes en el proceso de visualización, en el reconocimiento de reacciones } \\
\text { físicas y en las habilidades de autoeficacia de un entorno relajante. El contenido del vídeo presentó cuatro diferentes ambientes de relajación diseñados para ayudar a los } \\
\text { participantes a relajarse con un viaje de descubrimiento en cada entorno. }\end{array}$ \\
\hline $\begin{array}{l}\text { Terapia cognitivo conductual basa- } \\
\text { da en Internet versus terapia grupal } \\
\text { conductual cognitiva }\end{array}$ & Hedman et al. (2011) (1) & $\begin{array}{l}\text { ICBT: Una parte vital del tratamiento fue el acceso gradual al texto de autoayuda basado en Internet que comprende } 15 \text { módulos de texto. El paciente tuvo acceso a } \\
\text { un terapeuta a través de un sistema de mensajería segura en línea. La función del terapeuta era principalmente proporcionar información sobre el trabajo a domicilio y } \\
\text { otorgar acceso a los módulos de tratamiento. Sin embargo, el paciente puede comunicarse con el terapeuta en cualquier momento y esperar una respuesta dentro de las } 24 \\
\text { horas durante los días de semana. CBGT: La sesión individual preparó al participante para comenzar las sesiones de tratamiento grupal e incluyó una justificación para el } \\
\text { tratamiento grupal. }\end{array}$ \\
\hline TCC basada en Internet & Hedman et al. (2013) (2) & $\begin{array}{l}\text { El tratamiento consistió en un texto de autoayuda de } 118 \text { páginas provisto en } 12 \text { módulos semanales a través de una plataforma de tratamiento basada en Internet con } \\
\text { acceso a un terapeuta a través de un sistema de contacto en línea seguro. Los participantes tuvieron acceso a los módulos subsiguientes por su terapeuta. Cada módulo } \\
\text { estaba dedicado a un tema específico e incluía ejercicios de tarea. }\end{array}$ \\
\hline $\begin{array}{l}\text { TCC basada en internet y aplicación } \\
\text { de teléfono inteligente }\end{array}$ & Lindner et al. (2013) & $\begin{array}{l}\text { El programa de tratamiento contiene ocho módulos que cubren las conceptualizaciones de los trastornos de ansiedad de TCC y Terapia de Aceptación y Compromiso, así } \\
\text { como técnicas terapéuticas más específicas (reestructuración cognitiva, el entrenamiento de la exposición, ejercicios respiratorios, y prevención de recaídas). Se incluye } \\
\text { una aplicación de teléfono inteligente con los ejercicios de los módulos de tratamiento. }\end{array}$ \\
\hline $\begin{array}{l}\text { Aplicación de teléfono inteligente } \\
\text { basada en la terapia de aceptación y } \\
\text { compromiso }\end{array}$ & Ly et al. (2012) & La intervención consistió en una aplicación de teléfono inteligente y psicoeducación basada en la web. \\
\hline $\begin{array}{l}\text { IntelliCare: un conjunto de apli- } \\
\text { caciones }\end{array}$ & Mohr et al. (2017) & $\begin{array}{l}\text { IntelliCare conjunto de aplicaciones, cada una de las cuales admite una sola habilidad o utiliza un estilo interactivo único para apoyar la adquisición de una serie de } \\
\text { habilidades. El programa consiste en } 14 \text { aplicaciones en total, incluidas } 13 \text { aplicaciones clínicas diseñadas para mejorar los síntomas de depresión y ansiedad a través de } \\
\text { estrategias de tratamiento eficaces, y la aplicación "Hub", que coordina la experiencia de un usuario con las aplicaciones clínicas. }\end{array}$ \\
\hline $\begin{array}{l}\text { BiofeedBack, Realidad Virtual y } \\
\text { teléfono móvil }\end{array}$ & Pallavicini et al. (2009) & $\begin{array}{l}\text { Las sesiones del programa INTERPRIDE se centran la exposición de los pacientes a través de realidad virtual a escenarios virtuales (una hermosa isla tropical frente al } \\
\text { océano) siguiendo la narrativa registrada por el terapeuta basada en relajación muscular. El paciente está conectado con biosensores que registran sus parámetros fisiológi- } \\
\text { cos (conducta de la piel, frecuencia cardíaca, respiración). Se registra una medida de referencia de estos parámetros durante } 3 \text { minutos en condición de reposo. Siguiendo } \\
\text { un sendero que lo guía a través de la isla, el paciente llega al punto de inicio, donde los diferentes paneles indican las direcciones hacia las diferentes áreas objetivo. En } \\
\text { cada una de estas áreas se proporciona un ejercicio de relajación; durante este entrenamiento, siguiendo las indicaciones dadas por la guía de voz, el paciente trata de } \\
\text { relajarse. }\end{array}$ \\
\hline
\end{tabular}


Tabla 3. Descripción de los programas de intervención (continuación)

\begin{tabular}{|c|c|c|}
\hline INTERVENCIÓN & AUTORES & DESCRIPCIÓN DEL PROGRAMA \\
\hline TCC con soporte de teléfono & Per Carlbring et al. (2007) & $\begin{array}{l}\text { El tratamiento se basó en métodos cognitivo-conductuales establecidos. Cada módulo incluyó información, ejercicios y un cuestionario interactivo, y terminó con tres a } \\
\text { ocho preguntas de ensayo. Se les pidió a los participantes que explicaran con sus propias palabras las secciones más importantes del módulo que acababan de completar, } \\
\text { proporcionaron registros de pensamientos, su experiencia y el resultado de sus ejercicios de exposición. Para cada módulo, los participantes debían publicar al menos un } \\
\text { mensaje en un grupo de discusión en línea sobre un tema predeterminado. }\end{array}$ \\
\hline mHealth Game: "FLOWY" & Pham et al. (2016) & $\begin{array}{l}\text { Intervención "Flowy" es un juego de } m \text { Health que involucra a los usuarios en una serie de minijuegos donde utilizan ejercicios de reentrenamiento respiratorio y realizan } \\
\text { respiración diafragmática para aliviar la ansiedad. Los minijuegos de 'Flowy' presentan desde navegar en bote por un río hasta volar globos en el cielo. Los usuarios tocan } \\
\text { la pantalla con el dedo mientras inhalan y retiran el dedo de la pantalla mientras exhalan para controlar la mecánica del juego. }\end{array}$ \\
\hline SmartCAT: plataforma $\mathrm{mHealth}$ & Pramana et al. (2014) (1) & $\begin{array}{l}\text { SmartCAT (tratamiento de ansiedad infantil mejorada para teléfonos inteligentes) la aplicación indica a los pacientes que utilicen las habilidades aprendidas en una sesión } \\
\text { de TCC durante sus actividades diarias. }\end{array}$ \\
\hline $\begin{array}{l}\text { TCC y APP móvil } \\
\text { Proyecto SmarCAT }\end{array}$ & Pramana et al. (2018) (2) & $\begin{array}{l}\text { El sistema SmartCAT consiste en una aplicación de teléfono inteligente para niños y un portal clínico integrado. La aplicación contiene (1) una serie de juegos interacti- } \\
\text { vos y actividades para reforzar la comprensión de habilidades, (2) un entrenador de habilidades in vivo que indica al participante que use habilidades de terapia cognitiva } \\
\text { conductual durante experiencias emocionales del mundo real, (3) un desafío en el hogar módulo para fomentar las tareas de exposición en el hogar, (4) un sistema de re- } \\
\text { compensa digital que contiene puntos digitales y trofeos, y (5) una interfaz de mensajería de terapeuta-paciente. Los terapeutas utilizan un portal seguro basado en la Web } \\
\text { conectado a la aplicación para configurar las actividades requeridas para cada sesión, recibir o enviar mensajes, administrar los premios y desafíos de los participantes, y } \\
\text { ver datos y figuras que resumen el uso de la aplicación. }\end{array}$ \\
\hline $\begin{array}{l}\text { Teléfono móvil y programa a través } \\
\text { de la web. Proyecto MyCompass }\end{array}$ & Proudfoot et al. (2013) & $\begin{array}{l}\text { myCompass es una intervención de salud pública totalmente automatizada, de autoayuda, que se adapta al usuario y no tiene aportaciones del terapeuta. El autocontrol en } \\
\text { tiempo real de los síntomas a través del teléfono móvil y ordenador es una característica terapéutica clave. Los usuarios pueden controlar por sí mismos tres síntomas de } \\
\text { su elección en cualquier momento. Cada síntoma se califica en una escala de } 10 \text { puntos. }\end{array}$ \\
\hline $\begin{array}{l}\text { TCC basada en internet } \\
\text { "BRAVE" }\end{array}$ & Spence et al. (2011) & $\begin{array}{l}\text { "BRAVE" se dirige a cuatro tipos de ansiedad: ansiedad social, ansiedad generalizada, ansiedad por separación y fobias específicas. Las sesiones incluyen psicoeduca- } \\
\text { ción, entrenamiento de relajación, reconocimiento de los síntomas fisiológicos de la ansiedad, la exposición gradual, la resolución de problemas y el refuerzo personal. } \\
\text { Las sesiones para padres presentan estrategias de crianza para ayudar a los padres a ayudar a los adolescentes a implementar habilidades de manejo de la ansiedad. Se } \\
\text { utilizan gráficos, sonidos, juegos y cuestionarios llamativos para mantener el nivel de interés de los adolescentes. }\end{array}$ \\
\hline Mobile app & Stolz et al. (2018) & $\begin{array}{l}\text { Todos los participantes fueron guiados por entrenadores individuales durante su período activo. El tratamiento se dirige tanto a factores conductuales como cognitivos, } \\
\text { como atención autocentrada, pensamientos automáticos negativos, evitación experiencial, y síntomas de ansiedad cognitiva. El programa de autoayuda y la aplicación } \\
\text { para teléfonos inteligentes se estructuraron en ocho módulos. }\end{array}$ \\
\hline $\begin{array}{l}\text { Manejo del estrés con teléfonos } \\
\text { móviles }\end{array}$ & Villani et al. (2013) & $\begin{array}{l}\text { Protocolo de intervención apoyado por herramientas móviles y que corresponde a las necesidades reales de las enfermeras de oncología. En la condición experimental, } \\
\text { los participantes vieron ocho videoclips con una narrativa creada ad hoc utilizando teléfonos móviles. En la condición de control, los participantes vieron ocho videoclips } \\
\text { neutrales sin ningún tipo de narrativa. La música de fondo era la misma en ambas condiciones. Durante este tiempo, todos los participantes vieron ocho videos multime- } \\
\text { dia a través de diferentes medios, dos veces por semana. La adquisición de habilidades y la fase de ensayo se combinaron con dos tipos de técnicas de relajación: la Re- } \\
\text { lajación Muscular Progresiva (PMR; Jacobson, 1938) y el Entrenamiento Autógeno (AT; Schultz, 1977). Cada sesión estuvo compuesta por la presentación de un vídeo. } \\
\text { Los primeros seis clips de audio y vídeo para el grupo experimental se desarrollaron utilizando la plataforma "Just Leap In" y mostraron un entorno virtual relajante. }\end{array}$ \\
\hline $\begin{array}{l}\text { Programa Breathe: TCC basado en } \\
\text { Internet }\end{array}$ & Wozney et al. (2015) & $\begin{array}{l}\text { El programa Breathe consta de } 8 \text { módulos (Módulo } 1 \text { (Introducción a la respiración / Visión general de la ansiedad) Módulo } 2 \text { (Poder negativo de creencias poco } \\
\text { realistas) Módulo } 3 \text { (Trampas de pensamiento comunes) Módulo } 4 \text { (Estrategias de relajación y afrontamiento positivo). Módulo } 5 \text { (Evitar / Prepararse para enfrentar los } \\
\text { miedos) Módulo } 6 \text { (Escaleras del miedo / Planificación estratégica) Módulo } 7 \text { (Practicar actividades de exposición / Tratar con contratiempos) Módulo } 8 \text { (Manteniendo } \\
\text { ganancias). }\end{array}$ \\
\hline
\end{tabular}

